\title{
SOME LIMITING LAWS ASSOCIATED WITH THE INTEGRATED BROWNIAN MOTION *
}

\author{
Christophe Profeta ${ }^{1}$
}

\begin{abstract}
We study some limit theorems for the normalized law of integrated Brownian motion perturbed by several examples of functionals: the first passage time, the $n$th passage time, the last passage time up to a finite horizon and the supremum. We show that the penalization principle holds in all these cases and give descriptions of the conditioned processes. In particular, it is remarkable that the penalization by the $n$th passage time is independent of $n$, and always gives the same penalized process, i.e. integrated Brownian motion conditioned not to hit 0 . Our results rely on some explicit formulae obtained by Lachal and on enlargement of filtrations.
\end{abstract}

Mathematics Subject Classification. 60J65, 60G15, 60G44.

Received July 16, 2013. Revised December 11, 2013.

\section{INTRODUCTION}

The study of limiting laws or penalizations of a given process may be seen as a way to condition a probability law by an event of null probability, or by an a.s. infinite random variable. As a simple example, let for instance $\left(B_{t}, t \geq 0\right)$ be a Brownian motion started from $x>0,\left(\mathcal{F}_{t}=\sigma\left(B_{s}, s \leq t\right), t \geq 0\right)$ its natural filtration, and assume that one would like to define the law of $B$ conditioned to stay positive. Denoting the first hitting time of $B$ to level 0 by $\sigma_{0}=\inf \left\{t \geq 0, B_{t}=0\right\}$, one natural way to do this is to look at the possible limit, as $t \rightarrow+\infty$, of the following probability family:

$$
\mathbb{P}_{x}^{(t)}(\bullet)=\mathbb{P}_{x}\left(\bullet \mid \sigma_{0}>t\right) \quad(t \geq 0) .
$$

In this case, for any $\Lambda_{s} \in \mathcal{F}_{s}$, it is easily proven that:

$$
\mathbb{P}_{x}^{(t)}\left(\Lambda_{s}\right)=\frac{\mathbb{E}_{x}\left[1_{\Lambda_{s}} 1_{\left\{\sigma_{0}>t\right\}}\right]}{\mathbb{P}_{x}\left(\sigma_{0}>t\right)} \underset{t \rightarrow+\infty}{\longrightarrow} \mathbb{E}_{x}\left[1_{\Lambda_{s}} \frac{B_{s}}{x} 1_{\left\{\sigma_{0}>s\right\}}\right]
$$

Therefore, if $\mathbb{P}_{x}^{(\infty)}$ exists, then we must have:

$$
\mathbb{P}_{x \mid \mathcal{F}_{s}}^{(\infty)}=\frac{B_{s}}{x} 1_{\left\{\sigma_{0}>s\right\}} \cdot \mathbb{P}_{x \mid \mathcal{F}_{s}}
$$

Keywords and phrases. Integrated Brownian motion, penalization, passage times.

* This research benefited from the support of the "Chaire Marchés en Mutation", Fédération Bancaire Française.

1 Laboratoire de Mathématiques et Modélisation d'Evry (LaMME), Université d'Evry-Val-d'Essonne, UMR CNRS 8071,

91037 Evry cedex, France. christophe.profeta@univ-evry.fr 
and one recognizes here the absolute continuity formula between the Wiener measure and the law of a threedimensional Bessel process. More generally, replacing $\left(1_{\left\{\sigma_{0}>t\right\}}, t \geq 0\right)$ by a general weight $\left(\Gamma_{t}, t \geq 0\right)$, one may give the following definition of penalization:

Definition 1.1. Let $X$ be a stochastic process defined on a filtered probability space $\left(\Omega, \mathcal{F}_{\infty},\left(\mathcal{F}_{t}\right)_{t \geq 0}, \mathbb{P}\right)$ and let $\left(\Gamma_{t}, t \geq 0\right)$ be a measurable process taking positive values, and such that $0<\mathbb{E}\left[\Gamma_{t}\right]<\infty$ for any $t>0$. We say that the process $\left(\Gamma_{t}, t \geq 0\right)$ satisfies the penalization principle if there exists a probability measure $\mathbb{Q}^{(\Gamma)}$ defined on $\left(\Omega, \mathcal{F}_{\infty}\right)$ such that:

$$
\forall s \geq 0, \forall \Lambda_{s} \in \mathcal{F}_{s}, \quad \lim _{t \rightarrow+\infty} \frac{\mathbb{E}\left[1_{\Lambda_{s}} \Gamma_{t}\right]}{\mathbb{E}\left[\Gamma_{t}\right]}=\mathbb{Q}^{(\Gamma)}\left(\Lambda_{s}\right) .
$$

The systematic study of such problems started in 2006 with a series of papers by B. Roynette, P. Vallois and M. Yor (see the survey [26] and references within, or the monograph [27]) who look at the limiting laws of Brownian motion perturbed by different kinds of functionals: supremum, infimum, local time, length of excursion, additive functionals ... In [23], they managed to unify a large part of these penalizations in a general theorem where the three-dimensional Bessel process plays an important role. Some of their results were generalized to random walks [5], stable Lévy processes [30], and linear diffusions [24,28]. We shall study here some examples of penalizations of a non-Markov process, i.e. the integrated Brownian motion

$$
X_{t}=X_{0}+\int_{0}^{t} B_{u} \mathrm{~d} u
$$

where $\left(B_{u}, u \geq 0\right)$ is a standard Brownian motion. Of course, the process $(X, B)$ is Markov, with generator $\mathcal{G}$ given by

$$
\mathcal{G}=\frac{1}{2} \frac{\partial^{2}}{\partial y^{2}}+y \frac{\partial}{\partial x}
$$

and we denote by $\mathbb{P}_{(x, y)}$ its law when started from $(x, y)$. We assume that $(X, B)$ is defined on the canonical space $\Omega=\mathcal{C}^{1,0}\left(\mathbb{R}_{+} \rightarrow \mathbb{R}\right)$ (that is, the functions of class $\mathcal{C}^{1}$ in $x$ and $\mathcal{C}^{0}$ in $\left.y\right)$ and we denote by $\left(\mathcal{F}_{t}=\sigma\left(B_{s}, s \leq\right.\right.$ $t), t \geq 0$ ) the natural filtration generated by $B$, with $\mathcal{F}_{\infty}:=\bigvee_{t \geq 0} \mathcal{F}_{t}$.

There is already a large literature dealing with the integrated Brownian motion: we may refer for instance to McKean [21], Goldman [9], Lachal [15,17,19], and more recently Touboul \& Faugeras [29] for studies related to the passage times, to Lachal [20] for a detailed study of its excursions, or to Khoshnevisan and Shi [13] for small ball problems... Some of these results are summarized in the three notes by Lachal $[14,16,18]$.

It may also be noticed that the integrated Brownian motion, who was first introduced by Langevin in the early 1900s, is still topical today. In particular, some authors have recently studied several reflections of this process, see for instance Bertoin [3], the thesis of Jacob [10], or Bossy and Jabir [4].

Our aim in this paper is to show that, for the integrated Brownian motion, the penalization principle holds with several kinds of functionals. We shall nevertheless see that the behavior of the penalized process is sometimes very different than the one we might have expected when comparing with classical one-dimensional Markov processes.

The outline of the paper is as follows:

- We start, in Section 2, by first reviewing and further studying the penalization of integrated Brownian motion by the first hitting time of 0 .

- This result is then generalized in Section 3 where we study the penalization by the $n$th passage time at 0 . We prove in particular that this penalization actually does not depend on $n$.

- We next look at an intermediate approach in Section 4, and consider the penalization by the last passage time at 0 before a finite horizon. In this case, we shall see that the penalized process may cross the level 0 a few times before leaving it forever.

- Section 5 is dedicated to a brief account on the penalization by the running supremum.

- And finally we postpone till Section 5 some computational and rather technical proofs. 


\section{Preliminaries}

\subsection{Notations}

We start by introducing a few notation. Let $p_{t}(x, y ; u, v)$ be the transition density of $(X, B)$ :

$$
p_{t}(x, y ; u, v)=\frac{\sqrt{3}}{\pi t^{2}} \exp \left(-\frac{6(u-x)^{2}}{t^{3}}+\frac{6(u-x)(v+y)}{t^{2}}-\frac{2\left(v^{2}+v y+y^{2}\right)}{t}\right),
$$

and define the function $q_{t}(x, y ; u, v)$ by

$$
q_{t}(x, y ; u, v)=p_{t}(x, y ; u, v)-p_{t}(x, y ; u,-v) .
$$

Let $T_{0}$ be the first hitting time of 0 by the process $X$ after time 0 :

$$
T_{0}=\inf \left\{t>0 ; X_{t}=0\right\} .
$$

We are interested in the penalization of $X$ by the process $\left(1_{\left\{T_{0}>t\right\}}, t \geq 0\right)$, i.e. we would like to define the law of $X$ conditioned not to hit 0 . Such a study was already carried out by Groeneboom, Jongbloed and Wellner in [8], and we shall complete their results here.

Define the function $h:] 0,+\infty) \times \mathbb{R} \longmapsto \mathbb{R}^{+}$by:

$$
h(x, y)=\int_{0}^{+\infty} \int_{0}^{+\infty} w^{3 / 2} q_{s}(x, y ; 0,-w) \mathrm{d} s \mathrm{~d} w .
$$

This function is harmonic for the generator $\mathcal{G}$ (in the sense that $\mathcal{G} h=0$ ), and admits the following representation:

$$
h(x, y)= \begin{cases}x^{1 / 6}\left(\frac{2}{9}\right)^{1 / 6} \frac{y}{x^{1 / 3}} U\left(\frac{1}{6}, \frac{4}{3}, \frac{2}{9} \frac{y^{3}}{x}\right) & \text { if } y>0 \\ x^{1 / 6}\left(\frac{2}{9}\right)^{-1 / 6} \frac{\Gamma(1 / 3)}{\Gamma(1 / 6)} & \text { if } y=0 \\ -x^{1 / 6}\left(\frac{2}{9}\right)^{1 / 6} \frac{1}{6} \frac{y}{x^{1 / 3}} V\left(\frac{1}{6}, \frac{4}{3}, \frac{2}{9} \frac{y^{3}}{x}\right) & \text { if } y<0\end{cases}
$$

where $U$ denotes the confluent hypergeometric function defined on [1], Chapter 13, p. 504, and $V$ is given, for $z<0$ by:

$$
V\left(\frac{1}{6}, \frac{4}{3}, z\right)=\mathrm{e}^{z} U\left(\frac{7}{6}, \frac{4}{3},-z\right) .
$$

Since $U(a, b, z) \underset{z \rightarrow+\infty}{\sim} z^{-a}$, we deduce by letting $x \downarrow 0$ in the expression of $h$ that:

$$
h(0, y)=\lim _{x \downarrow 0} h(x, y)=\sqrt{y^{+}} \quad \text { where } y^{+}=y \vee 0=\max (y, 0) .
$$

The asymptotics of the survival function of $T_{0}$ is given in the following theorem.

Theorem 2.1 [8]. For every $x>0$ and $y \in \mathbb{R}$, or $x=0$ and $y>0$, there is the asymptotics:

$$
\mathbb{P}_{(x, y)}\left(T_{0}>t\right) \underset{t \rightarrow+\infty}{\sim} \frac{3 \Gamma(1 / 4)}{2^{3 / 4} \pi^{3 / 2}} \frac{h(x, y)}{t^{1 / 4}} .
$$

\section{Remark 2.2.}

- This result was first established by Goldman in the case $y=0$, see [9]. Some generalizations were then obtained by Izosaki \& Kotani [11] who compute the asymptotics of the survival function of $T_{0}$ for the process

$$
X_{t}=x+\int_{0}^{t}\left(B_{s}^{\beta} 1_{\left\{B_{s}>0\right\}}-\frac{\left|B_{s}\right|^{\beta}}{c} 1_{\left\{B_{s}<0\right\}}\right) \mathrm{d} s \quad\left(c>0 \text { and } \beta>-\frac{3}{2}\right) .
$$


- The rate of decay $t^{-1 / 4}$ is actually one of the few explicit persistence exponents that are known, see the recent survey on this subject by Aurzada and Simon [2].

- We shall generalize this result in the next section, where we will determine the asymptotics of the $n$th passage time of $X$ at level 0 .

From Theorem 2.1, we deduce in particular that $x \longrightarrow h(x, y)$ and $y \longrightarrow h(x, y)$ are increasing functions. Observe also that $h$ admits the scaling property:

$$
h(x, y)=x^{1 / 6} h\left(1, \frac{y}{x^{1 / 3}}\right)
$$

and that there exist two constants $a$ and $b$ such that:

$$
h(x, y) \leq a x^{1 / 6}+b \sqrt{|y|} .
$$

\subsection{Penalization with the first passage time}

To prove penalization results, we shall, as is usual, rely on the following meta-theorem:

Theorem 2.3. Let $\left(\Gamma_{t}, t \geq 0\right)$ be a measurable process taking positive values and such that $0<\mathbb{E}\left[\Gamma_{t}\right]<\infty$ for every $t>0$. Assume that for any $s \geq 0$ :

$$
\lim _{t \rightarrow+\infty} \frac{\mathbb{E}\left[\Gamma_{t} \mid \mathcal{F}_{s}\right]}{\mathbb{E}\left[\Gamma_{t}\right]}=M_{s}
$$

exists a.s. and satisfies:

$$
\mathbb{E}\left[M_{s}\right]=1 .
$$

Then:

(i) For any $s \geq 0$ and $\Lambda_{s} \in \mathcal{F}_{s}$ :

$$
\lim _{t \rightarrow+\infty} \frac{\mathbb{E}\left[1_{\Lambda_{s}} \Gamma_{t}\right]}{\mathbb{E}\left[\Gamma_{t}\right]}=\mathbb{E}\left[M_{s} 1_{\Lambda_{s}}\right]
$$

that is, the convergence also holds in $L^{1}(\Omega)$.

(ii) $\left(M_{s}, s \geq 0\right)$ is a martingale.

(iii) There exists a probability measure $\mathbb{Q}$ on $\left(\Omega, \mathcal{F}_{\infty}\right)$ such that, for any $s>0$ and $\Lambda_{s} \in \mathcal{F}_{s}$ :

$$
\mathbb{Q}\left(\Lambda_{s}\right)=\mathbb{E}\left[M_{s} 1_{\Lambda_{s}}\right] .
$$

Proof. The proof of this theorem is classical: point (i) follows from Scheffe's lemma, point (ii) is a direct consequence of point (i) and point (iii) follows from Kolmogorov's existence theorem since the family of probabilities $\left(\mathbb{P}^{(t)}:=M_{t} \cdot \mathbb{P}_{\mid \mathcal{F}_{t}}, t \geq 0\right)$ is consistent, see $([27]$, Sect. 0.3, p. 8) for a discussion on this theorem.

We now state the main result of this section, which is essentially a reformulation of a result of [8]. Let $\bar{p}_{t}$ denote the transition density of the process $(X, B)$ killed when $X$ hits 0 :

$$
\bar{p}_{t}(x, y ; u, v) \mathrm{d} u \mathrm{~d} v=\mathbb{P}_{(x, y)}\left(X_{t} \in \mathrm{d} u, B_{t} \in \mathrm{d} v ; t<T_{0}\right) .
$$

Theorem 2.4. Let $x>0$ and $y \in \mathbb{R}$ or $x=0$ and $y>0$.

(i) For every $s \geq 0$ and $\Lambda_{s} \in \mathcal{F}_{s}$, we have:

$$
\lim _{t \rightarrow+\infty} \mathbb{E}_{(x, y)}\left[\frac{1_{\left\{T_{0}>t\right\}}}{\mathbb{P}_{(x, y)}\left(T_{0}>t\right)} 1_{\Lambda_{s}}\right]=\mathbb{E}_{(x, y)}\left[\frac{h\left(X_{s}, B_{s}\right)}{h(x, y)} 1_{\left\{T_{0}>s\right\}} 1_{\Lambda_{s}}\right] .
$$


(ii) There exists a probability measure $\mathbb{Q}_{(x, y)}$ defined on $\left(\Omega, \mathcal{F}_{\infty}\right)$ such that

$$
\forall s \geq 0, \forall \Lambda_{s} \in \mathcal{F}_{s}, \quad \mathbb{Q}_{(x, y)}\left(\Lambda_{s}\right)=\mathbb{E}_{(x, y)}\left[\frac{h\left(X_{s}, B_{s}\right)}{h(x, y)} 1_{\left\{T_{0}>s\right\}} 1_{\Lambda_{s}}\right] .
$$

(iii) Under $\mathbb{Q}_{(x, y)}$, the process $\left(X_{t}, t \geq 0\right)$ is a.s. transient and never hits 0:

$$
\mathbb{Q}_{(x, y)}\left(\lim _{t \rightarrow+\infty} X_{t}=+\infty\right)=1 \quad \text { and } \quad \mathbb{Q}_{(x, y)}\left(T_{0}=+\infty\right)=1 .
$$

(iv) The coordinate process $(X, B)$ under $\mathbb{Q}_{(x, y)}$ has the same law as the solution of the system of SDEs:

$$
\begin{cases}d X_{t}=B_{t} \mathrm{~d} t & X_{0}=x \\ \mathrm{~d} B_{t}=\mathrm{d} W_{t}+\frac{1}{h\left(X_{t}, B_{t}\right)} \frac{\partial h}{\partial y}\left(X_{t}, B_{t}\right) \mathrm{d} t & B_{0}=y\end{cases}
$$

where $\left(W_{t}, t \geq 0\right)$ is a $\mathbb{Q}_{(x, y)}$-Brownian motion. Its transition density is given by:

$$
\mathbb{Q}_{(x, y)}\left(X_{t} \in \mathrm{d} u, B_{t} \in \mathrm{d} v\right)=\frac{1}{h(x, y)} \bar{p}_{t}(x, y ; u, v) h(u, v) \mathrm{d} u \mathrm{~d} v .
$$

Proof. Applying the Markov property:

$$
\mathbb{P}_{(x, y)}\left(T_{0}>t \mid \mathcal{F}_{s}\right)=1_{\left\{T_{0}>s\right\}} \mathbb{P}_{\left(X_{s}, B_{s}\right)}\left(T_{0}>t-s\right) \underset{t \rightarrow+\infty}{\sim} \frac{3 \Gamma(1 / 4)}{2^{3 / 4} \pi^{3 / 2}} \frac{h\left(X_{s}, B_{s}\right)}{t^{1 / 4}} 1_{\left\{T_{0}>s\right\}}
$$

hence, we have the a.s. convergence:

$$
\lim _{t \rightarrow+\infty} \frac{\mathbb{P}_{(x, y)}\left(T_{0}>t \mid \mathcal{F}_{s}\right)}{\mathbb{P}_{(x, y)}\left(T_{0}>t\right)}=\frac{h\left(X_{s}, B_{s}\right)}{h(x, y)} 1_{\left\{T_{0}>s\right\}}
$$

and the convergence in $L^{1}(\Omega)$ as well as the existence of $\mathbb{Q}_{(x, y)}$ will follow from Theorem 2.3 once we have proven that

$$
\forall s \geq 0, \quad \mathbb{E}_{(x, y)}\left[h\left(X_{s}, B_{s}\right) 1_{\left\{s<T_{0}\right\}}\right]=h(x, y) .
$$

But it is known from [8] that the function

$$
(u, v) \longrightarrow \frac{1}{h(x, y)} \bar{p}_{t}(x, y ; u, v) h(u, v)
$$

is a probability density on $[0,+\infty) \times \mathbb{R}$. Therefore,

$$
\mathbb{E}_{(x, y)}\left[h\left(X_{t}, B_{t}\right) 1_{\left\{t<T_{0}\right\}}\right]=\int_{0}^{+\infty} \int_{\mathbb{R}} h(u, v) \bar{p}_{t}(x, y ; u, v) \mathrm{d} u \mathrm{~d} v=h(x, y)
$$

which is the desired result. Next, the fact that $X$ a.s. never hits 0 is immediate since:

$$
\mathbb{Q}_{(x, y)}\left(T_{0}=\infty\right)=\lim _{t \rightarrow+\infty} \mathbb{Q}_{(x, y)}\left(T_{0}>t\right)=\lim _{t \rightarrow+\infty} \frac{\mathbb{E}_{(x, y)}\left[h\left(X_{t}, B_{t}\right) 1_{\left\{T_{0}>t\right\}}\right]}{h(x, y)}=\lim _{t \rightarrow+\infty} 1=1 .
$$

To prove that $X$ is transient, observe that the process $\left(N_{t}=\frac{1}{h\left(X_{t}, B_{t}\right)}, t \geq 0\right)$ is a positive $\mathbb{Q}_{(x, y)}$-local martingale which therefore converges $\mathbb{Q}_{(x, y)}$-a.s. toward a r.v. $N_{\infty}$. But, as $\mathbb{Q}_{(x, y)}\left[N_{t}\right]=\frac{1}{h(x, y)} \mathbb{P}_{(x, y)}\left(T_{0}>t\right) \underset{t \rightarrow+\infty}{\longrightarrow} 0$, we deduce that $N_{\infty}=0$, which, given the behavior of $h$ at infinity, implies that $X_{t} \underset{t \rightarrow+\infty}{\longrightarrow}+\infty \mathbb{Q}_{(x, y)}$-a.s. 
Finally, to study the law of $(X, B)$ under $\mathbb{Q}_{(x, y)}$, we shall apply Girsanov's theorem. Indeed, on the set $\left\{t<T_{0}\right\}$, Itô's formula yields, since $h$ is harmonic for $\mathcal{G}$ :

$$
\ln \left(h\left(X_{t}, B_{t}\right)\right)=\int_{0}^{t} \frac{1}{h\left(X_{s}, B_{s}\right)} \frac{\partial h}{\partial y}\left(X_{s}, B_{s}\right) \mathrm{d} B_{s}-\frac{1}{2} \int_{0}^{t} \frac{1}{h^{2}\left(X_{s}, B_{s}\right)}\left(\frac{\partial h}{\partial y}\left(X_{s}, B_{s}\right)\right)^{2} \mathrm{~d} s
$$

and, since the process

$$
\begin{aligned}
Z_{t \wedge T_{0}} & =\exp \left(\int_{0}^{t \wedge T_{0}} \frac{1}{h\left(X_{s}, B_{s}\right)} \frac{\partial h}{\partial y}\left(X_{s}, B_{s}\right) \mathrm{d} B_{s}-\frac{1}{2} \int_{0}^{t \wedge T_{0}} \frac{1}{h^{2}\left(X_{s}, B_{s}\right)}\left(\frac{\partial h}{\partial y}\left(X_{s}, B_{s}\right)\right)^{2} \mathrm{~d} s\right) \\
& =h\left(X_{t}, B_{t}\right) 1_{\left\{t<T_{0}\right\}} \quad\left(\text { since } \mathbb{P}_{(x, y)}\left(B_{T_{0}}<0\right)=1\right)
\end{aligned}
$$

is a true martingale, Girsanov's theorem implies that the process $(X, B)$ under $\mathbb{Q}_{(x, y)}$ is a weak solution of the system $(2.5)$ on the set $\left\{t<T_{0}\right\}$. But as $\mathbb{Q}_{(x, y)}\left(T_{0}=+\infty\right)=1$, we conclude that the coordinate process $(X, B)$ under $\mathbb{Q}_{(x, y)}$ is a solution of $(2.5)$ on $[0,+\infty)$.

Remark 2.5. The direct proof of the existence and uniqueness of the solution of the system (2.5) is not straightforward since the coefficients do not satisfy the usual Lipchitz and growth conditions. Nevertheless, it is proven in [8] by a localization argument that (2.5) admits a unique strong solution for $x>0$ and $y \in \mathbb{R}$. If $x=0$ and $y>0$, we may also construct a unique strong solution as follows: define $\sigma_{\varepsilon}=\inf \left\{t \geq 0, B_{t}=\varepsilon\right\}$ and assume that $\varepsilon$ is such that $y>\varepsilon$. The function

$$
(u, v) \longmapsto \frac{1}{h(u, v \vee \varepsilon)} \frac{\partial h}{\partial v}(u, v \vee \varepsilon)=\frac{1}{v \vee \varepsilon}-\frac{1}{9} \frac{(v \vee \varepsilon)^{2}}{u} \frac{U\left(\frac{7}{6}, \frac{7}{3}, \frac{2}{9} \frac{(v \vee \varepsilon)^{3}}{u}\right)}{U\left(\frac{1}{6}, \frac{4}{3}, \frac{2}{9} \frac{(v \vee \varepsilon)^{3}}{u}\right)}
$$

is globally Lipschitz, so we may construct a unique strong solution of (2.5) up to time $\sigma_{\varepsilon}$. By pasting this solution with the solution of the system started from $\left(X_{\sigma_{\varepsilon}}, \varepsilon\right)$ (which is known to exist since $X_{\sigma_{\varepsilon}}>0$ a.s.), we obtain a solution of $(2.5)$ on $[0,+\infty)$. Furthermore, from $([12]$, Thm. 4.20, p. 322), since the coefficients are bounded on compact subsets, this solution is strongly Markovian.

\subsection{Passage times of integrated Brownian motion conditioned to be positive}

As mentioned earlier, the solution of the system (2.5) was studied in [8], where the authors prove in particular that the first component $X$ is transient and goes towards $+\infty \mathbb{Q}_{(x, y)}$-a.s. We shall complete their results by computing some first and last passage times, thanks to the weak absolute continuity formula. Let $T_{a}=\inf \{t>$ $\left.0, X_{t}=a\right\}$ and $\sigma_{b}=\inf \left\{t \geq 0, B_{t}=b\right\}$.

Theorem 2.6. Let $x>0$ and $y \in \mathbb{R}$.

(i) For $x>a$, the density of the couple $\left(T_{a}, B_{T_{a}}\right)$ under $\mathbb{Q}_{(x, y)}$ is given by

$$
\mathbb{Q}_{(x, y)}\left(T_{a} \in \mathrm{d} t, B_{T_{a}} \in \mathrm{d} z\right)=\frac{h(a, z)}{h(x, y)} \mathbb{P}_{(x, y)}\left(T_{a} \in \mathrm{d} t, B_{T_{a}} \in \mathrm{d} z\right) .
$$

(ii) For $y \geq b \geq 0$, the density of the couple $\left(\sigma_{b}, X_{\sigma_{b}}\right)$ under $\mathbb{Q}_{(x, y)}$ is given by:

$$
\mathbb{Q}_{(x, y)}\left(\sigma_{b} \in \mathrm{d} t, X_{\sigma_{b}} \in \mathrm{d} z\right)=\frac{h(z, b)}{h(x, y)} \mathbb{P}_{(x, y)}\left(\sigma_{b} \in \mathrm{d} t, X_{\sigma_{b}} \in \mathrm{d} z\right) .
$$


Proof. The proof is straightforward and relies on Doob's stopping theorem:

$$
\begin{aligned}
\mathbb{Q}_{(x, y)}\left(T_{a}>t, B_{T_{a}}<z\right) & =\frac{1}{h(x, y)} \mathbb{E}_{(x, y)}\left[h\left(X_{t}, B_{t}\right) 1_{\left\{T_{a} \wedge T_{0}>t, B_{T_{a} \wedge T_{0}}<z\right\}}\right] \\
& =\frac{1}{h(x, y)} \mathbb{E}_{(x, y)}\left[h\left(a, B_{T_{a}}\right) 1_{\left\{T_{a}>t, B_{T_{a}}<z\right\}}\right]
\end{aligned}
$$

as, for $x>a$, the continuity of paths implies that $T_{a}<T_{0}$. Similarly, for $y \geq b \geq 0$, we must have $\sigma_{b}<T_{0}$ :

$$
\mathbb{Q}_{(x, y)}\left(\sigma_{b}>t, X_{\sigma_{b}}<z\right)=\frac{1}{h(x, y)} \mathbb{E}_{(x, y)}\left[h\left(X_{\sigma_{b}}, b\right) 1_{\left\{\sigma_{b}>t, X_{\sigma_{b}}<z\right\}}\right] .
$$

Remark 2.7. The same proof applies when $x=a$ and $y>0$ since in this case, the process $\left(X_{t}, t \geq 0\right)$ is strictly increasing in the neighborhood of $X_{0}=x$. Using the explicit formula of McKean [21], this leads to a simple expression:

$$
\mathbb{Q}_{(a, y)}\left(T_{a} \in \mathrm{d} t, B_{T_{a}} \in \mathrm{d} z\right)=\frac{h(a, z)}{h(a, y)} \frac{3|z|}{t^{2} \pi \sqrt{2}} \exp \left(-\frac{2}{t}\left(y^{2}-y|z|+z^{2}\right)\right)\left(\int_{0}^{4 y|z| / s} \frac{1}{\sqrt{\pi \theta}} \mathrm{e}^{-\frac{3}{2} \theta} d \theta\right) 1_{]-\infty, 0]}(z) \mathrm{d} t \mathrm{~d} z
$$

Corollary 2.8. Let $x>a \geq 0$ :

$$
\mathbb{Q}_{(x, y)}\left(T_{a}<+\infty\right)=1-\frac{h(x-a, y)}{h(x, y)} .
$$

The proof of this corollary is a direct consequence of the following lemma:

Lemma 2.9. Let $x>a$. Then:

$$
\mathbb{E}_{(x, y)}\left[h\left(a, B_{T_{a}}\right)\right]=h(x, y)-h(x-a, y) .
$$

Proof. It does not seem easy to compute directly this expression using the explicit distribution of $B_{T_{a}}$ (see Lachal [15]), so we shall rather rely on a martingale argument. From Doob's stopping theorem, since $T_{a}<T_{0}$ a.s.:

$$
h(x, y)=\mathbb{E}_{(x, y)}\left[h\left(X_{t \wedge T_{a}}, B_{t \wedge T_{a}}\right) 1_{\left\{t \wedge T_{a}<T_{0}\right\}}\right]=\mathbb{E}_{(x, y)}\left[h\left(a, B_{T_{a}}\right) 1_{\left\{T_{a}<t\right\}}\right]+\mathbb{E}_{(x, y)}\left[h\left(X_{t}, B_{t}\right) 1_{\left\{t<T_{a}\right\}}\right]
$$

hence, passing to the limit:

$$
\mathbb{E}_{(x, y)}\left[h\left(a, B_{T_{a}}\right)\right]=h(x, y)-\lim _{t \rightarrow+\infty} \mathbb{E}_{(x, y)}\left[h\left(X_{t}, B_{t}\right) 1_{\left\{t<T_{a}\right\}}\right]
$$

Now, by translation,

$$
\mathbb{E}_{(x, y)}\left[h\left(X_{t}, B_{t}\right) 1_{\left\{t<T_{a}\right\}}\right]=\mathbb{E}_{(x-a, y)}\left[h\left(a+X_{t}, B_{t}\right) 1_{\left\{t<T_{0}\right\}}\right]
$$

and we shall compare this term with $h(x-a, y)$, which, from (2.4), may be written:

$$
h(x-a, y)=\mathbb{E}_{(x-a, y)}\left[h\left(X_{t}, B_{t}\right) 1_{\left\{t<T_{0}\right\}}\right] .
$$

Then recall that the functions $x \rightarrow h(x, y)$ and $y \rightarrow h(x, y)$ are increasing, so we need to study:

$$
\begin{aligned}
\left|\mathbb{E}_{(x, y)}\left[h\left(X_{t}, B_{t}\right) 1_{\left\{t<T_{a}\right\}}\right]-h(x-a, y)\right| & =\mathbb{E}_{(x-a, y)}\left[h\left(a+X_{t}, B_{t}\right) 1_{\left\{t<T_{0}\right\}}\right]-\mathbb{E}_{(x-a, y)}\left[h\left(X_{t}, B_{t}\right) 1_{\left\{t<T_{0}\right\}}\right] \\
& =\int_{0}^{+\infty} \int_{\mathbb{R}}(h(a+u, v)-h(u, v)) \bar{p}_{t}(x-a, y ; u, v) \mathrm{d} u \mathrm{~d} v .
\end{aligned}
$$


Let $A>0$ be fixed. We first write:

$$
\int_{0}^{A} \int_{\mathbb{R}}(h(a+u, v)-h(u, v)) \bar{p}_{t}(x-a, y ; u, v) \mathrm{d} u \mathrm{~d} v \leq \int_{0}^{A} \int_{\mathbb{R}}(h(a+A, v)-h(0, v)) \bar{p}_{t}(x-a, y ; u, v) \mathrm{d} u \mathrm{~d} v .
$$

Now, observe that the function

$$
v \longrightarrow h(a+A, v)-h(0, v)
$$

is bounded by a constant, say $K$. Indeed, it is a positive and continuous function whose limits at $+\infty$ and $-\infty$ both equal 0 . Therefore,

$$
\begin{aligned}
\int_{0}^{A} \int_{\mathbb{R}}(h(a+A, v)-h(0, v)) \bar{p}_{t}(x-a, y ; u, v) \mathrm{d} u \mathrm{~d} v & \leq K \int_{0}^{A} \int_{\mathbb{R}} \bar{p}_{t}(x-a, y ; u, v) \mathrm{d} u \mathrm{~d} v \\
& \leq K \mathbb{P}_{(x-a, y)}\left(T_{0}>t\right) \underset{t \rightarrow+\infty}{\longrightarrow} 0 .
\end{aligned}
$$

Now, adding and subtracting $(a+u)^{1 / 6} h\left(1, \frac{v}{u^{1 / 3}}\right)$, we decompose the remaining integral in two terms:

$$
\int_{A}^{+\infty} \int_{\mathbb{R}}\left((a+u)^{1 / 6} h\left(1, \frac{v}{(a+u)^{1 / 3}}\right)-u^{1 / 6} h\left(1, \frac{v}{u^{1 / 3}}\right)\right) \bar{p}_{t}(x-a, y ; u, v) \mathrm{d} u \mathrm{~d} v=I_{t}^{(1)}+I_{t}^{(2)}
$$

with

$$
\left\{\begin{array}{l}
I_{t}^{(1)}=\int_{A}^{+\infty} \int_{\mathbb{R}}(a+u)^{1 / 6}\left(h\left(1, \frac{v}{(a+u)^{1 / 3}}\right)-h\left(1, \frac{v}{u^{1 / 3}}\right)\right) \bar{p}_{t}(x-a, y ; u, v) \mathrm{d} u \mathrm{~d} v, \\
I_{t}^{(2)}=\int_{A}^{+\infty} \int_{\mathbb{R}}\left((a+u)^{1 / 6}-u^{1 / 6}\right) h\left(1, \frac{v}{u^{1 / 3}}\right) \bar{p}_{t}(x-a, y ; u, v) \mathrm{d} u \mathrm{~d} v .
\end{array}\right.
$$

(i) The second term may be dealt with easily:

$$
\begin{aligned}
I_{t}^{(2)} & =\int_{A}^{+\infty} \int_{\mathbb{R}}\left(\left(\frac{a}{u}+1\right)^{1 / 6}-1\right) h(u, v) \bar{p}_{t}(x-a, y ; u, v) \mathrm{d} u \mathrm{~d} v \\
& =h(x-a, y) \mathbb{Q}_{(x-a, y)}\left[\left(\left(\frac{a}{X_{t}}+1\right)^{1 / 6}-1\right) 1_{\left\{X_{t} \geq A\right\}}\right] \underset{t \rightarrow+\infty}{\longrightarrow} 0
\end{aligned}
$$

from the dominated convergence theorem, since $\mathbb{Q}_{(x-a, y)}\left(\lim _{t \rightarrow+\infty} X_{t}=+\infty\right)=1$.

(ii) As for the first term, we further make the decomposition

$$
I_{t}^{(1)}=\int_{A}^{+\infty} \int_{0}^{+\infty} \ldots \mathrm{d} u \mathrm{~d} v+\int_{A}^{+\infty} \int_{-\infty}^{0} \ldots \mathrm{d} u \mathrm{~d} v .
$$

Observe that, since $y \longmapsto h(1, y)$ is increasing, the first integral (on the right-hand side) is negative, while the second one is positive. But, as

$$
I_{t}^{(1)}+I_{t}^{(2)} \geq 0
$$

it is enough to bound $I_{t}^{(1)}$ from above by the positive one. We thus write:

$$
\begin{aligned}
I_{t}^{(1)} & \leq \int_{A}^{+\infty} \int_{-\infty}^{0}(a+u)^{1 / 6}\left(h\left(1, \frac{v}{(a+u)^{1 / 3}}\right)-h\left(1, \frac{v}{u^{1 / 3}}\right)\right) \bar{p}_{t}(x-a, y ; u, v) \mathrm{d} u \mathrm{~d} v \\
& =\int_{A}^{+\infty} \int_{-\infty}^{0}(a+u)^{1 / 6}\left(h\left(1, \frac{v}{u^{1 / 3}} \frac{u^{1 / 3}}{(a+u)^{1 / 3}}\right)-h\left(1, \frac{v}{u^{1 / 3}}\right)\right) \bar{p}_{t}(x-a, y ; u, v) \mathrm{d} u \mathrm{~d} v \\
& \leq \int_{A}^{+\infty} \int_{-\infty}^{0}(a+u)^{1 / 6}\left(h\left(1, \frac{v}{u^{1 / 3}} \frac{1}{\left(\frac{a}{A}+1\right)^{1 / 3}}\right)-h\left(1, \frac{v}{u^{1 / 3}}\right)\right) \bar{p}_{t}(x-a, y ; u, v) \mathrm{d} u \mathrm{~d} v .
\end{aligned}
$$


Let $\varepsilon>0$. Since $\lim _{z \rightarrow-\infty} h(1, z)=0$, there exists $-b<0$ such that

$$
\forall z \leq-b, \quad h\left(1, \frac{z}{2}\right) \leq \frac{\varepsilon}{2}
$$

In particular, if $A$ is such that

$$
\frac{1}{\left(\frac{a}{A}+1\right)^{1 / 3}} \geq \frac{1}{2}
$$

then, for $z \leq-b$ :

$$
\left|h\left(1, \frac{z}{\left(\frac{a}{A}+1\right)^{1 / 3}}\right)-h(1, z)\right| \leq h\left(1, \frac{z}{2}\right)+h(1, z) \leq \varepsilon
$$

Next, from Heine's theorem, the function $z \longrightarrow h(1, z)$ is uniformly continuous in the compact set $[-b, 0]$. Therefore, there exists $\eta>0$ such that

$$
\forall(y, z) \in[-b, 0]^{2}, \quad|z-y| \leq \eta \Longrightarrow|h(1, z)-h(1, y)| \leq \varepsilon .
$$

Thus, if we take $y=\frac{z}{\left(\frac{a}{A}+1\right)^{1 / 3}}$ and assume that $A$ is chosen large enough such that

$$
|b|\left(1-\frac{1}{\left(\frac{a}{A}+1\right)^{1 / 3}}\right) \leq \eta,
$$

we deduce that, for every $z \in[-b, 0]$, we also have:

$$
\left|h\left(1, \frac{z}{\left(\frac{a}{A}+1\right)^{1 / 3}}\right)-h(1, z)\right| \leq \varepsilon .
$$

Now, going back to (2.6), we may write:

$$
\begin{aligned}
I_{t}^{(1)} & \leq \varepsilon \int_{A}^{+\infty} \int_{-\infty}^{0}(a+u)^{1 / 6} \bar{p}_{t}(x-a, y ; u, v) \mathrm{d} u \mathrm{~d} v \\
& \leq \varepsilon\left(\frac{a}{A}+1\right)^{1 / 6} \int_{A}^{+\infty} \int_{-\infty}^{0} u^{1 / 6} \bar{p}_{t}(x-a, y ; u, v) \mathrm{d} u \mathrm{~d} v \\
& \leq \varepsilon\left(\frac{a}{A}+1\right)^{1 / 6} \mathbb{E}_{(x-a, y)}\left[X_{t}^{1 / 6} 1_{\left\{t<T_{0}\right\}} 1_{\left\{B_{t} \leq 0\right\}}\right] \\
& =\varepsilon\left(\frac{a}{A}+1\right)^{1 / 6} \mathbb{E}_{(x-a, y)}\left[X_{t}^{1 / 6} 1_{\left\{t<T_{0}\right\}} \mid B_{t} \leq 0\right] \mathbb{P}_{(x, y)}\left(B_{t} \leq 0\right)
\end{aligned}
$$

Observe next that, by decomposing

$$
X_{t}=x+\int_{0}^{\gamma_{0}^{(t)}} B_{s} \mathrm{~d} s+\int_{\gamma_{0}^{(t)}}^{t} B_{s} \mathrm{~d} s \quad \text { and } \quad\left\{t<T_{0}\right\}=\left\{\inf _{u \leq t}\left(x+\int_{0}^{\gamma_{0}^{(t)} \wedge u} B_{s} \mathrm{~d} s+\int_{\gamma_{0}^{(t)} \wedge u}^{u} B_{s} \mathrm{~d} s\right)>0\right\}
$$


with $\gamma_{0}^{(t)}=\sup \left\{s \leq t, B_{s}=0\right\}$, we deduce that:

$$
\begin{aligned}
I_{t}^{(1)} & \leq \varepsilon\left(\frac{a}{A}+1\right)^{1 / 6} \mathbb{E}_{(x-a, y)}\left[X_{t}^{1 / 6} 1_{\left\{t<T_{0}\right\}} \mid B_{t} \geq 0\right] \mathbb{P}_{(x, y)}\left(B_{t} \leq 0\right) \\
& =\varepsilon\left(\frac{a}{A}+1\right)^{1 / 6} \mathbb{E}_{(x-a, y)}\left[X_{t}^{1 / 6} 1_{\left\{t<T_{0}\right\}} 1_{\left\{B_{t} \geq 0\right\}}\right] \frac{\mathbb{P}_{(x, y)}\left(B_{t} \leq 0\right)}{\mathbb{P}_{(x, y)}\left(B_{t} \geq 0\right)} \\
& \leq \varepsilon\left(\frac{a}{A}+1\right)^{1 / 6} \mathbb{E}_{(x-a, y)}\left[\frac{h\left(X_{t}, B_{t}\right)}{h(1,0)} 1_{\left\{t<T_{0}\right\}} 1_{\left\{B_{t} \geq 0\right\}}\right] \frac{\mathbb{P}_{(x, y)}\left(B_{t} \leq 0\right)}{\mathbb{P}_{(x, y)}\left(B_{t} \geq 0\right)} \\
& \leq \varepsilon\left(\frac{a}{A}+1\right)^{1 / 6} \frac{h(x-a, y)}{h(1,0)} \frac{\mathbb{P}_{(x, y)}\left(B_{t} \leq 0\right)}{\mathbb{P}_{(x, y)}\left(B_{t} \geq 0\right)} \\
& \underset{t \rightarrow+\infty}{\longrightarrow}\left(\frac{a}{A}+1\right)^{1 / 6} \frac{h(x-a, y)}{h(1,0)}
\end{aligned}
$$

where we have used that:

$$
x^{1 / 6}=\frac{h(x, 0)}{h(1,0)} \leq \frac{h(x, y)}{h(1,0)} \quad \text { for } y \geq 0 .
$$

Theorem 2.10. Let $x>0$ and $y \in \mathbb{R}$ or $x=0$ and $y>0$. The cumulative distribution function of the last passage time of $X$ at level a under $\mathbb{Q}_{(x, y)}$ is given by:

$$
\mathbb{Q}_{(x, y)}\left(g_{a}<t\right)=\frac{1}{h(x, y)} \int_{\mathbb{R}} \int_{0}^{+\infty} h(u, v) \bar{p}_{t}(x, y ; u+a, v) \mathrm{d} u \mathrm{~d} v
$$

with $g_{a}=\sup \left\{t \geq 0, X_{t}=a\right\}$.

Proof. Using the Markov property and Corollary 2.8:

$$
\begin{aligned}
\mathbb{Q}_{(x, y)}\left(g_{a}<t\right) & =\mathbb{Q}_{(x, y)}\left(\left\{g_{a}<t\right\} \cap\left\{X_{t}>a\right\}\right) \\
& =\int_{a}^{+\infty} \int_{\mathbb{R}} \mathbb{Q}_{(x, y)}\left(g_{a}<t \mid X_{t}=u, B_{t}=v\right) \mathbb{Q}_{(x, y)}\left(X_{t} \in \mathrm{d} u, B_{t} \in \mathrm{d} v\right) \\
& =\frac{1}{h(x, y)} \int_{a}^{+\infty} \int_{\mathbb{R}} \mathbb{Q}_{(x, y)}\left(T_{a} \circ \theta_{t}=+\infty \mid X_{t}=u, B_{t}=v\right) \bar{p}_{t}(x, y ; u, v) h(u, v) \mathrm{d} u \mathrm{~d} v \\
& =\frac{1}{h(x, y)} \int_{a}^{+\infty} \int_{\mathbb{R}} \mathbb{Q}_{(u, v)}\left(T_{a}=+\infty\right) \bar{p}_{t}(x, y ; u, v) h(u, v) \mathrm{d} u \mathrm{~d} v \\
& =\frac{1}{h(x, y)} \int_{a}^{+\infty} \int_{\mathbb{R}} h(u-a, y) \bar{p}_{t}(x, y ; u, v) \mathrm{d} u \mathrm{~d} v \\
& =\frac{1}{h(x, y)} \int_{0}^{+\infty} \int_{\mathbb{R}} h(u, y) \bar{p}_{t}(x, y ; u+a, v) \mathrm{d} u \mathrm{~d} v .
\end{aligned}
$$

\section{Penalization with the $n$ Th passage time}

Let $T_{0}^{(n)}$ be the $n$th passage time of $X$ at the level 0 :

$$
T_{0}^{(0)}=0 \quad \text { and } \quad T_{0}^{(n)}=\inf \left\{t>T_{0}^{(n-1)} ; X_{t}=0\right\} .
$$


We are now interested in the penalization of $X$ by the process $\left(1_{\left\{T_{0}^{(n)}>t\right\}}, t \geq 0\right)$. To this end, we first need to compute some asymptotics:

Proposition 3.1. Let $b \neq 0$. There is the asymptotics, for $n \geq 1$ :

$$
\mathbb{P}_{(0, b)}\left(T_{0}^{(n)}>t\right) \underset{t \rightarrow+\infty}{\sim} \frac{2^{1 / 4} \Gamma(1 / 4) \sqrt{|b|}}{\sqrt{\pi}(n-1) !}\left(\frac{9}{4 \pi^{2}}\right)^{n / 2} \frac{(\ln (t))^{n-1}}{t^{1 / 4}} .
$$

The proof of this result is given in Section 5 as it is purely computational and rather technical.

Remark 3.2. The asymptotics behavior in $n$ was already studied by McKean in [21] (with a slightly different definition of $T_{0}^{(n)}$ ) where he derives the following results:

$$
\ln \left(T_{0}^{(n)}\right) \underset{n \rightarrow+\infty}{\sim} \frac{8 \pi}{\sqrt{3}} n \quad \text { and } \quad \ln \left|B_{T_{0}^{(n)}}\right| \underset{n \rightarrow+\infty}{\sim} \frac{4 \pi}{\sqrt{3}} n
$$

Theorem 3.3. Let $x>0$ and $y \in \mathbb{R}$.

(i) There is the asymptotics:

$$
\mathbb{P}_{(x, y)}\left(T_{0}^{(n)}>t\right) \underset{t \rightarrow+\infty}{\sim} \frac{2^{1 / 4} \Gamma(1 / 4)}{\sqrt{\pi}(n-1) !}\left(\frac{9}{4 \pi^{2}}\right)^{n / 2} \frac{(\ln (t))^{n-1}}{t^{1 / 4}} h(x, y) .
$$

(ii) For every $s \geq 0$ and $\Lambda_{s} \in \mathcal{F}_{s}$, we have:

$$
\lim _{t \rightarrow+\infty} \mathbb{E}_{(x, y)}\left[\frac{1_{\Lambda_{s}} 1_{\left\{T_{0}^{(n)}>t\right\}}}{\mathbb{P}_{(x, y)}\left(T_{0}^{(n)}>t\right)}\right]=\mathbb{E}_{(x, y)}\left[\frac{h\left(X_{s}, B_{s}\right)}{h(x, y)} 1_{\left\{T_{0}>s\right\}} 1_{\Lambda_{s}}\right] .
$$

Therefore, the penalization by the $\mathrm{n}$ th passage time leads to the same conditioned process as the penalization by the first passage time.

Remark 3.4. This behavior is very different from the classical one-dimensional Markov processes which have already been studied in the literature. Indeed, in all the known examples (random walks, Brownian motion, recurrent diffusions ...) the penalization by the $n$th passage time (or by its analogue, the inverse local time) gives penalized processes which highly depend on $n$, see $[5,25,28]$.

Proof. The proof of point (i) follows from Proposition 3.1 and from the Markov property. Indeed, from the formula (see $[8,15]$ ),

$$
\mathbb{P}_{(x, y)}\left(T_{0} \in \mathrm{d} s, B_{T_{0}} \in \mathrm{d} z\right) /(\mathrm{d} s \mathrm{~d} z)=|z|\left(q_{s}(x, y ; 0, z)-\int_{0}^{s} \int_{0}^{+\infty} q_{s-u}(x, y ; 0, w) \mathbb{P}_{(0, z)}\left(T_{0} \in \mathrm{d} u, B_{T_{0}} \in \mathrm{d} w\right)\right) 1_{\{z<0\}}
$$

we may write:

$$
\begin{aligned}
& \mathbb{P}_{(x, y)}\left(T_{0}^{(n)}>t\right)-\mathbb{P}_{(x, y)}\left(T_{0}>t\right) \\
& =\int_{0}^{t} \int_{-\infty}^{0} \mathbb{P}_{(0, z)}\left(T_{0}^{(n-1)}>t-s\right) \mathbb{P}_{(x, y)}\left(T_{0} \in \mathrm{d} s, B_{T_{0}} \in \mathrm{d} z\right) \\
& =\int_{0}^{t} \mathrm{~d} s \int_{-\infty}^{0} \mathrm{~d} z \mathbb{P}_{(0, z)}\left(T_{0}^{(n-1)}>t-s\right)|z|\left(q_{s}(x, y ; 0, z)-\int_{0}^{s} \int_{0}^{+\infty} q_{s-u}(x, y ; 0, w) \mathbb{P}_{(0, z)}\left(T_{0} \in \mathrm{d} u, B_{T_{0}} \in \mathrm{d} w\right)\right)
\end{aligned}
$$


We now take the Laplace transform of both sides:

$$
\begin{aligned}
& \int_{0}^{+\infty} \mathrm{e}^{-\lambda t} \mathbb{P}_{(x, y)}\left(T_{0}^{(n)}>t\right) \mathrm{d} t-\int_{0}^{+\infty} \mathrm{e}^{-\lambda t} \mathbb{P}_{(x, y)}\left(T_{0}>t\right) \mathrm{d} t \\
& =\int_{-\infty}^{0} \int_{0}^{+\infty}\left(\int_{0}^{+\infty} \mathrm{e}^{-\lambda t} \mathbb{P}_{(0, z)}\left(T_{0}^{(n-1)}>t\right) \mathrm{d} t\right)\left(\int_{0}^{+\infty} \mathrm{e}^{-\lambda t} q_{t}(x, y ; 0, w) \mathrm{d} t\right) \mathbb{E}_{(0, z)}\left[\mathrm{e}^{-\lambda T_{0}}, B_{T_{0}} \in \mathrm{d} w\right] z \mathrm{~d} z \\
& \quad-\int_{-\infty}^{0}\left(\int_{0}^{+\infty} \mathrm{e}^{-\lambda t} \mathbb{P}_{(0, z)}\left(T_{0}^{(n-1)}>t\right) \mathrm{d} t\right)\left(\int_{0}^{+\infty} \mathrm{e}^{-\lambda t} q_{t}(x, y ; 0, z) \mathrm{d} t\right) z \mathrm{~d} z .
\end{aligned}
$$

But, from the symmetry relation, for $\omega<0<z$ :

$$
\mathbb{E}_{(0, z)}\left[\mathrm{e}^{-\lambda T_{0}}, B_{T_{0}} \in \mathrm{d} w\right] \mathrm{d} z=-\frac{w}{z} \mathbb{E}_{(0, w)}\left[\mathrm{e}^{-\lambda T_{0}}, B_{T_{0}} \in \mathrm{d} z\right] \mathrm{d} w
$$

and the fact that $q_{t}(x, y ; u, v)=-q_{t}(x, y ; u,-v)$, we deduce that:

$$
\begin{aligned}
\int_{0}^{+\infty} & \mathrm{e}^{-\lambda t} \mathbb{P}_{(x, y)}\left(T_{0}^{(n)}>t\right) \mathrm{d} t-\int_{0}^{+\infty} \mathrm{e}^{-\lambda t} \mathbb{P}_{(x, y)}\left(T_{0}>t\right) \mathrm{d} t \\
= & \int_{0}^{+\infty}\left(\int_{0}^{+\infty} \mathrm{e}^{-\lambda t}\left(\mathbb{P}_{(0, w)}\left(T_{0}^{(n)}>t\right)-\mathbb{P}_{(0, w)}\left(T_{0}>t\right)\right) \mathrm{d} t\right)\left(\int_{0}^{+\infty} \mathrm{e}^{-\lambda t} q_{t}(x, y ; 0,-w) \mathrm{d} t\right) w \mathrm{~d} w \\
& -\int_{-\infty}^{0}\left(\int_{0}^{+\infty} \mathrm{e}^{-\lambda t} \mathbb{P}_{(0, z)}\left(T_{0}^{(n-1)}>t\right) \mathrm{d} t\right)\left(\int_{0}^{+\infty} \mathrm{e}^{-\lambda t} q_{t}(x, y ; 0, z) \mathrm{d} t\right) z \mathrm{~d} z .
\end{aligned}
$$

Next, from Proposition 3.1 and from the Tauberian Theorem ([7], Chap. XIII-5, p. 446),

$$
\int_{0}^{+\infty} \mathrm{e}^{-\lambda t} \mathbb{P}_{(0, w)}\left(T_{0}^{(n)}>t\right) \mathrm{d} t \underset{\lambda \rightarrow 0}{\sim} \frac{2^{1 / 4} \Gamma(1 / 4) \sqrt{|w|}}{\sqrt{\pi}(n-1) !}\left(\frac{9}{4 \pi^{2}}\right)^{n / 2} \Gamma(5 / 4) \frac{(-\ln (\lambda))^{n-1}}{\lambda^{5 / 4}}
$$

hence, taking only the leading terms on each side of the equality, we obtain:

$$
\begin{aligned}
& \int_{0}^{+\infty} \mathrm{e}^{-\lambda t} \mathbb{P}_{(x, y)}\left(T_{0}^{(n)}\right.>t) \mathrm{d} t \\
& \underset{\lambda \rightarrow 0}{\sim} \frac{2^{1 / 4} \Gamma(1 / 4) \Gamma(5 / 4)}{\sqrt{\pi}(n-1) !}\left(\frac{9}{4 \pi^{2}}\right)^{n / 2} \frac{(-\ln (\lambda))^{n-1}}{\lambda^{5 / 4}} \int_{0}^{+\infty} \sqrt{|w|}\left(\int_{0}^{+\infty} q_{t}(x, y ; 0,-w) \mathrm{d} t\right) w \mathrm{~d} w
\end{aligned}
$$

and the result follows from another application of the Tauberian theorem.

To prove point (ii), we finally write, applying the Markov property:

$$
\begin{aligned}
\mathbb{P}_{(x, y)}\left(T_{0}^{(n)}>t \mid \mathcal{F}_{s}\right)=1_{\left\{T_{0}>s\right\}} \mathbb{P}_{\left(X_{s}, B_{s}\right)}\left(T_{0}^{(n)}>t-s\right)+1_{\left\{T_{0} \leq s, T_{0}^{(2)}>s\right\}} & \mathbb{P}_{\left(X_{s}, B_{s}\right)}\left(T_{0}^{(n-1)}>t-s\right) \\
& +\ldots+1_{\left\{T_{0}^{(n-1)} \leq s, T_{0}^{(n)}>s\right\}} \mathbb{P}_{\left(X_{s}, B_{s}\right)}\left(T_{0}>t-s\right),
\end{aligned}
$$

and, due to Point $(i)$, the leading asymptotics comes from the first term $1_{\left\{T_{0}>s\right\}} \mathbb{P}_{\left(X_{s}, B_{s}\right)}\left(T_{0}^{(n)}>t-s\right)$.

\section{Penalization with the last passage time up to a Finite horizon}

We have seen that the penalization with the $n$th passage time gives a process which never hit 0 . We shall try to obtain an intermediate penalization by choosing as weight process a function of the last passage time up to a finite horizon.

We define:

$$
\begin{aligned}
g_{0}^{(t)} & =\sup \left\{u \leq t ; X_{u}=0\right\} \\
& \left(=0 \quad \text { if }\left\{u \leq t ; X_{u}=0\right\}=\emptyset\right) .
\end{aligned}
$$


Lemma 4.1. The density of $g_{0}^{(t)}$ is given, for $\left.s \in\right] 0, t[$ by:

$$
\mathbb{P}_{(x, y)}\left(g_{0}^{(t)} \in \mathrm{d} s\right) / \mathrm{d} s=\int_{\mathbb{R}}|z| p_{s}(x, y ; 0, z) \mathbb{P}_{(0, z)}\left(T_{0}>t-s\right) \mathrm{d} z
$$

For $s=0$ :

$$
\mathbb{P}_{(x, y)}\left(g_{0}^{(t)}=0\right)=\mathbb{P}_{(x, y)}\left(T_{0}>t\right) .
$$

This lemma may be seen as a consequence of the main result of [17] where the author computes the joint law of $g_{0}^{(t)}, B_{g_{0}^{(t)}}$ and the first time the process $X$ hits 0 after the time $t$. For the sake of completeness, we give here a simple proof relying on a time reversal argument.

Proof. By time reversal (see [20], Lem. 2.12), we have:

$$
\mathbb{P}_{(x, y)}\left(g_{0}^{(t)}>s, X_{t} \in \mathrm{d} u, B_{t} \in \mathrm{d} v\right) /(\mathrm{d} u \mathrm{~d} v)=\mathbb{P}_{(u, v)}^{*}\left(T_{0}<t-s, X_{t} \in \mathrm{d} x, B_{t} \in \mathrm{d} y\right) /(\mathrm{d} x \mathrm{~d} y)
$$

where $\mathbb{P}^{*}$ denotes the law of the dual process of $(X, B)$, whose distribution equals that of $(X,-B)$. In particular, we have

$$
p_{s}^{*}(0, v ; x, y)=p_{s}(x, y ; 0, v)
$$

and

$$
\mathbb{P}_{(u, v)}^{*}\left(T_{0} \in \mathrm{d} s, B_{T_{0}} \in \mathrm{d} z\right)=|z| \bar{p}_{s}(0, z ; u, v) \mathrm{d} s \mathrm{~d} z .
$$

Then, applying the Markov property:

$$
\begin{aligned}
\mathbb{P}_{(u, v)}^{*}\left(T_{0}<t-s, X_{t} \in \mathrm{d} x, B_{t} \in \mathrm{d} y\right) /(\mathrm{d} x \mathrm{~d} y) & =\mathbb{E}_{(u, v)}^{*}\left[1_{\left\{T_{0}<t-s\right\}} p_{t-T_{0}}^{*}\left(0, B_{T_{0}} x, y\right)\right] \\
& =\mathbb{E}_{(u, v)}^{*}\left[1_{\left\{T_{0}<t-s\right\}} p_{t-T_{0}}\left(0, B_{T_{0}},-x, y\right)\right] \\
& =\int_{0}^{t-s} \int_{\mathbb{R}} p_{t-r}(x, y ; 0, z)|z| \bar{p}_{r}(0, z ; u, v) \mathrm{d} r \mathrm{~d} z
\end{aligned}
$$

and the result follows by differentiation with respect to $s$.

Let $\varphi: \mathbb{R}^{+} \longrightarrow \mathbb{R}^{+}$be a continuous function with compact support, $\varphi \neq 0$. We set

$$
\Phi(x, y)=\varphi(0) h(x, y) 1_{\{x \geq 0\}}+\varphi(0) h(-x,-y) 1_{\{x \leq 0\}}+\int_{\mathbb{R}}|z|^{3 / 2} \int_{0}^{+\infty} \varphi(s) p_{s}(x, y ; 0, z) \mathrm{d} s \mathrm{~d} z .
$$

Note that when $x=0$, one of the two symmetric terms is always null. Then we may state the following theorem:

\section{Theorem 4.2.}

(i) The process

$$
M_{t}^{\varphi}=\varphi\left(g_{0}^{(t)}\right)\left(h\left(X_{t}, B_{t}\right) 1_{\left\{X_{t} \geq 0\right\}}+h\left(-X_{t},-B_{t}\right) 1_{\left\{X_{t} \leq 0\right\}}\right)+\int_{\mathbb{R}}|z|^{3 / 2} \int_{0}^{+\infty} \varphi(t+s) p_{s}\left(X_{t}, B_{t} ; 0, z\right) \mathrm{d} s \mathrm{~d} z
$$

is a positive martingale which converges toward 0 as $t \rightarrow+\infty$.

(ii) Let $s \geq 0$ and $(x, y) \in \mathbb{R}^{2}$. For any $\Lambda_{s} \in \mathcal{F}_{s}$, we have:

$$
\lim _{t \rightarrow+\infty} \frac{\mathbb{E}_{(x, y)}\left[1_{\Lambda_{s}} \varphi\left(g_{0}^{(t)}\right)\right]}{\mathbb{E}_{(x, y)}\left[\varphi\left(g_{0}^{(t)}\right)\right]}=\mathbb{E}_{(x, y)}\left[1_{\Lambda_{s}} \frac{M_{s}^{\varphi}}{\Phi(x, y)}\right]
$$


(iii) There exists a family of probabilities $\left(\mathbb{Q}_{(x, y)}^{\varphi},(x, y) \in \mathbb{R}^{2}\right)$ such that, for any $t \geq 0$ :

$$
\mathbb{Q}_{(x, y) \mid \mathcal{F}_{t}}^{\varphi}=\frac{M_{t}^{\varphi}}{\Phi(x, y)} \cdot \mathbb{P}_{(x, y) \mid \mathcal{F}_{t}} .
$$

(iv) Let $g_{0}=\sup \left\{u \geq 0 ; X_{u}=0\right\}$. Then, $\mathbb{Q}_{(x, y)}^{\varphi}\left(g_{0}<+\infty\right)=1$ and conditionally on $g_{0}$ and $B_{g_{0}}$ :

(i) the processes $\left(X_{u}, u \leq g_{0}\right)$ and $\left(X_{u+g_{0}}, u \geq 0\right)$ are independent,

(ii) the process $\left(X_{u+g_{0}}, u \geq 0\right)$ has the same law as integrated Brownian motion started from $\left(0, B_{g_{0}}\right)$ and conditioned to stay positive if $B_{g_{0}}>0$, or conditioned to stay negative if $B_{g_{0}}<0$.

Proof. From Lemma 4.1, we have:

$$
\mathbb{E}_{(x, y)}\left[\varphi\left(g_{0}^{(t)}\right)\right]=\varphi(0) \mathbb{P}_{(x, y)}\left(T_{0}>t\right)+\int_{0}^{t} \mathrm{~d} s \varphi(s) \int_{\mathbb{R}}|z| p_{s}(x, y ; 0, z) \mathbb{P}_{(0, z)}\left(T_{0}>t-s\right) \mathrm{d} z
$$

and the asymptotics

$$
\begin{aligned}
& \mathbb{E}_{(x, y)}\left[\varphi\left(g_{0}^{(t)}\right)\right] \underset{t \rightarrow+\infty}{\sim} \frac{3 \Gamma(1 / 4)}{2^{3 / 4} \pi^{3 / 2} t^{1 / 4}}\left(\varphi(0) h(x, y) 1_{\{x>0\} \cup\{x=0, y>0\}}+\varphi(0) h(-x,-y) 1_{\{x<0\} \cup\{x=0, y<0\}}\right. \\
&\left.+\int_{\mathbb{R}}|z| h(0,|z|) \mathrm{d} z \int_{0}^{+\infty} \varphi(s) p_{s}(x, y ; 0, z) \mathrm{d} s\right) \\
& \sim \frac{3 \Gamma(1 / 4)}{t \rightarrow+\infty}{\frac{2}{3 / 4} \pi^{3 / 2} t^{1 / 4}}^{\sim} \Phi(x, y) .
\end{aligned}
$$

Applying the Markov property:

$$
\mathbb{E}_{(x, y)}\left[\varphi\left(g_{0}^{(t)}\right) \mid \mathcal{F}_{s}\right]=\mathbb{E}_{\left(X_{s}, B_{s}\right)}\left[\varphi\left(s+g_{0}^{(t-s)}\right)\right]
$$

so we obtain, thanks to (4.2) and to Theorem 2.1, the a.s. convergence:

$$
\lim _{t \rightarrow+\infty} \frac{\mathbb{E}_{(x, y)}\left[\varphi\left(g_{0}^{(t)}\right) \mid \mathcal{F}_{s}\right]}{\mathbb{E}_{(x, y)}\left[\varphi\left(g_{0}^{(t)}\right)\right]}=\frac{M_{s}^{\varphi}}{\Phi(x, y)} .
$$

To apply the Meta-Theorem 2.3, we need to prove that:

$$
\mathbb{E}_{(x, y)}\left[M_{t}^{\varphi}\right]=\Phi(x, y) .
$$

Thanks to Lemma 4.1, we have:

$$
\begin{aligned}
& \mathbb{E}_{(x, y)}\left[\varphi\left(g_{0}^{(t)}\right) h\left(X_{t}, B_{t}\right) 1_{\left\{X_{t} \geq 0\right\}}\right] \\
& =\varphi(0) \mathbb{E}_{(x, y)}\left[h\left(X_{t}, B_{t}\right) 1_{\left\{X_{t} \geq 0\right\}} 1_{\left\{t<T_{0}\right\}}\right]+\int_{0}^{t} \int_{0}^{+\infty} \int_{\mathbb{R}} \varphi(s) h(u, v) \\
& \quad \times\left(\int_{\mathbb{R}}|z| p_{s}(0, z ;-x, y) \bar{p}_{t-s}(0, z ; u, v) \mathrm{d} z\right) \mathrm{d} s \mathrm{~d} u \mathrm{~d} v \\
& =\varphi(0) h(x, y) 1_{\{x>0\} \cup\{x=0, y>0\}}+\int_{0}^{t} \int_{\mathbb{R}} \mathbb{E}_{(0, z)}\left[h\left(X_{t-s}, B_{t-s}\right) 1_{\left\{T_{0}>t-s\right\}} 1_{\left\{X_{t-s} \geq 0\right\}}\right] \varphi(s) p_{s}(0, z ;-x, y)|z| \mathrm{d} s \mathrm{~d} z \\
& =\varphi(0) h(x, y) 1_{\{x \geq 0\}}+\int_{0}^{t} \int_{\mathbb{R}} \sqrt{z^{+}} \varphi(s) p_{s}(0, z ;-x, y)|z| \mathrm{d} s \mathrm{~d} z \\
& =\varphi(0) h(x, y) 1_{\{x \geq 0\}}+\int_{0}^{t} \int_{0}^{+\infty} z^{3 / 2} \varphi(s) p_{s}(x, y ; 0, z) \mathrm{d} s \mathrm{~d} z .
\end{aligned}
$$


Similarly,

$$
\mathbb{E}_{(x, y)}\left[\varphi\left(g_{0}^{(t)}\right) h\left(-X_{t},-B_{t}\right) 1_{\left\{X_{t} \leq 0\right\}}\right]=\varphi(0) h(-x,-y) 1_{\{x \leq 0\}}+\int_{0}^{t} \int_{-\infty}^{0}|z|^{3 / 2} \varphi(s) p_{s}(x, y ; 0, z) \mathrm{d} s \mathrm{~d} z,
$$

and thus:

$$
\begin{aligned}
\mathbb{E}_{(x, y)}\left[\varphi\left(g_{0}^{(t)}\right) h\left(X_{t}, B_{t}\right) 1_{\left\{X_{t} \geq 0\right\}}+\varphi\left(g_{0}^{(t)}\right) h\left(-X_{t},-B_{t}\right) 1_{\left\{X_{t} \leq 0\right\}}\right] \\
=\varphi(0) h(x, y) 1_{\{x \geq 0\}}+\varphi(0) h(-x,-y) 1_{\{x \leq 0\}}+\int_{0}^{t} \int_{\mathbb{R}}|z|^{3 / 2} \varphi(s) p_{s}(x, y ; 0, z) \mathrm{d} s \mathrm{~d} z
\end{aligned}
$$

Next, the Chapman-Kolmogorov equation yields:

$$
\mathbb{E}_{(x, y)}\left[p_{s}\left(X_{t}, B_{t} ; 0, z\right)\right]=\iint_{\mathbb{R}^{2}} p_{s}(u, v ; 0, z) p_{t}(x, y ; u, v) \mathrm{d} u \mathrm{~d} v=p_{t+s}(x, y ; 0, z)
$$

hence

$$
\int_{\mathbb{R}}|z|^{3 / 2} \mathrm{~d} z \int_{0}^{+\infty} \varphi(s+t) \mathbb{E}_{(x, y)}\left[p_{s}\left(X_{t}, B_{t} ; 0, z\right)\right] \mathrm{d} s=\int_{t}^{+\infty} \varphi(s) \mathrm{d} s \int_{\mathbb{R}}|z|^{3 / 2} p_{s}(x, y ; 0, z) \mathrm{d} z
$$

and the desired result (4.3) follows by adding equations (4.4) and (4.5).

To prove Point $i v)$, we shall follow the ideas of Roynette, Vallois and Yor [25] and use enlargements of filtration. Let $g_{0}=\sup \left\{u \geq 0 ; X_{u}=0\right\}$ and define the (progressively) enlarged filtration $\left(\mathcal{G}_{t}, t \geq 0\right)$ to be the smallest filtration containing $\left(\mathcal{F}_{t}, t \geq 0\right)$ and such that $g_{0}$ is a $\left(\mathcal{G}_{t}\right)$-stopping time. From [22], if $W$ is a $\left(\mathbb{Q}_{(x, y)}^{\varphi},\left(\mathcal{F}_{t}\right)\right)$-Brownian motion, we have the decomposition:

$$
W_{t}=W_{t}^{\left(g_{0}\right)}+\int_{0}^{t \wedge g_{0}} \frac{d\left\langle Z^{\varphi}, W\right\rangle_{u}}{Z_{u}^{\varphi}}-\int_{t \wedge g_{0}}^{t} \frac{d\left\langle Z^{\varphi}, W\right\rangle_{u}}{1-Z_{u}^{\varphi}}
$$

where $\left(W_{t}^{\left(g_{0}\right)}, t \geq 0\right)$ is a $\left(\mathbb{Q}_{(x, y)}^{\varphi},\left(\mathcal{G}_{t}\right)\right)$-Brownian motion and $\left(Z_{t}^{\varphi}, t \geq 0\right)$ denotes Azéma's supermartingale:

$$
Z_{t}^{\varphi}=\mathbb{Q}_{(x, y)}^{\varphi}\left(g_{0}>t \mid \mathcal{F}_{t}\right)
$$

We now compute $Z^{\varphi}$ in our setting. Let $\sigma_{t}=\inf \left\{s>t, X_{s}=0\right\}$. We have $\left\{g_{0}>t\right\}=\left\{\sigma_{t}<+\infty\right\}$, so for $\Lambda_{t} \in \mathcal{F}_{t}$ :

$$
\begin{aligned}
\mathbb{Q}_{(x, y)}^{\varphi}\left(\Lambda_{t} \cap\left\{g_{0}>t\right\}\right) & =\mathbb{Q}_{(x, y)}^{\varphi}\left(\Lambda_{t} \cap\left\{\sigma_{t}<+\infty\right\}\right) \\
& =\lim _{n \rightarrow+\infty} \mathbb{Q}_{(x, y)}^{\varphi}\left(\Lambda_{t} \cap\left\{\sigma_{t}<t+n\right\}\right) \\
& =\lim _{n \rightarrow+\infty} \mathbb{E}_{(x, y)}\left[1_{\Lambda_{t} \cap\left\{\sigma_{t}<t+n\right\}} M_{\sigma_{t}}^{\varphi}\right] / \Phi(x, y) \\
& =\mathbb{E}_{(x, y)}\left[1_{\Lambda_{t}}\left(\varphi\left(\sigma_{t}\right) h\left(0,\left|B_{\sigma_{t}}\right|\right)+\int_{\mathbb{R}}|z|^{3 / 2} \mathrm{~d} z \int_{0}^{+\infty} \varphi\left(\sigma_{t}+s\right) p_{s}\left(0, B_{\sigma_{t}} ; 0, z\right) \mathrm{d} s\right)\right] / \Phi(x, y) .
\end{aligned}
$$

Conditioning with respect to $\mathcal{F}_{t}$, we obtain, on the one hand:

$$
\mathbb{E}_{(x, y)}\left[\varphi\left(\sigma_{t}\right) h\left(0,\left|B_{\sigma_{t}}\right|\right) \mid \mathcal{F}_{t}\right]=\mathbb{E}_{\left(X_{t}, B_{t}\right)}\left[\varphi\left(t+T_{0}\right) h\left(0,\left|B_{T_{0}}\right|\right)\right]
$$


Now, from Lachal [15], replacing $\left(X_{t}, B_{t}\right)$ by $(x, y)$ and using a symmetry argument:

$$
\begin{aligned}
\mathbb{E}_{(x, y)}[ & \left.\varphi\left(t+T_{0}\right) h\left(0,\left|B_{T_{0}}\right|\right) 1_{\left\{B_{T_{0}} \leq 0\right\}}\right]=\int_{0}^{+\infty} \int_{-\infty}^{0} \varphi(t+s) h(0,|z|)|z| \\
& \left(p_{s}(x, y ; 0, z)-\int_{0}^{s} \int_{0}^{+\infty} p_{s-u}(x, y ; 0,-w) \mathbb{P}_{(0,-|z|)}\left(T_{0} \in \mathrm{d} u, B_{T_{0}} \in \mathrm{d} w\right)\right) \mathrm{d} s \mathrm{~d} z \\
= & \int_{0}^{+\infty} \int_{-\infty}^{0} \varphi(t+s)|z|^{3 / 2} p_{s}(x, y ; 0, z) \mathrm{d} s \mathrm{~d} z \\
& -\int_{0}^{+\infty} \int_{-\infty}^{0} \varphi(t+s)|z|^{3 / 2} \int_{0}^{s} \int_{0}^{+\infty} p_{s-u}(0, w ; 0, z) \mathbb{P}_{(x, y)}\left(T_{0} \in \mathrm{d} u, B_{T_{0}} \in \mathrm{d} w\right) \mathrm{d} s \mathrm{~d} z \\
= & \int_{0}^{+\infty} \int_{-\infty}^{0} \varphi(t+s)|z|^{3 / 2} p_{s}(x, y ; 0, z) \mathrm{d} s \mathrm{~d} z \\
& -\int_{0}^{+\infty} \int_{-\infty}^{0} \varphi(t+s)|z|^{3 / 2} \mathbb{E}_{(x, y)}\left[1_{\left\{T_{0}<s\right\}} p_{s-T_{0}}\left(0, B_{T_{0}} ; 0, z\right)\right] \mathrm{d} s \mathrm{~d} z
\end{aligned}
$$

while:

$$
\begin{aligned}
\mathbb{E}_{(x, y)}\left[\varphi\left(t+T_{0}\right) h\left(0,\left|B_{T_{0}}\right|\right) 1_{\left\{B_{T_{0}}>0\right\}}\right]= & \int_{0}^{+\infty} \int_{0}^{+\infty} \varphi(t+s)|z|^{3 / 2} p_{s}(x, y ; 0, z) \mathrm{d} s \mathrm{~d} z \\
& -\int_{0}^{+\infty} \int_{0}^{+\infty} \varphi(t+s)|z|^{3 / 2} \mathbb{E}_{(x, y)}\left[1_{\left\{T_{0}<s\right\}} p_{s-T_{0}}\left(0, B_{T_{0}} ; 0, z\right)\right] \mathrm{d} s \mathrm{~d} z .
\end{aligned}
$$

On the other hand, by Fubini,

$$
\begin{aligned}
\int_{\mathbb{R}}|z|^{3 / 2} \mathrm{~d} z \int_{0}^{+\infty} \mathbb{E}_{(x, y)}\left[\varphi\left(\sigma_{t}+u\right) p_{u}\left(0, B_{\sigma_{t}} ; 0, z\right) \mid \mathcal{F}_{t}\right] \mathrm{d} u \\
=\int_{\mathbb{R}}|z|^{3 / 2} \mathrm{~d} z \int_{0}^{+\infty} \mathbb{E}_{\left(X_{t}, B_{t}\right)}\left[\varphi\left(t+T_{0}+u\right) p_{u}\left(0, B_{T_{0}} ; 0, z\right)\right] \mathrm{d} u \\
=\int_{\mathbb{R}}|z|^{3 / 2} \mathrm{~d} z \int_{0}^{+\infty} \varphi(t+s) \mathbb{E}_{\left(X_{t}, B_{t}\right)}\left[1_{\left\{T_{0}<s\right\}} p_{s-T_{0}}\left(0, B_{T_{0}} ; 0, z\right)\right] \mathrm{d} s .
\end{aligned}
$$

Summing (4.7), (4.8) and (4.9) gives the expression of Azéma's supermartingale:

$$
Z_{t}^{\varphi}=\mathbb{Q}_{(x, y)}^{\varphi}\left(g_{0}>t \mid \mathcal{F}_{t}\right)=\frac{1}{M_{t}^{\varphi}} \int_{\mathbb{R}}|z|^{3 / 2} \mathrm{~d} z \int_{0}^{+\infty} \varphi(t+s) p_{s}\left(X_{t}, B_{t} ; 0, z\right) \mathrm{d} s=\frac{N_{t}^{\varphi}}{M_{t}^{\varphi}}
$$

where we set $N_{t}^{\varphi}=\int_{\mathbb{R}}|z|^{3 / 2} \mathrm{~d} z \int_{0}^{+\infty} \varphi(t+s) p_{s}\left(X_{t}, B_{t} ; 0, z\right) \mathrm{d} s$. Observe besides that

$$
\mathbb{Q}_{(x, y)}^{\varphi}\left(g_{0}>t\right)=\mathbb{Q}_{(x, y)}^{\varphi}\left[Z_{t}^{\varphi}\right]=\frac{1}{\Phi(x, y)} \mathbb{E}_{(x, y)}\left[N_{t}^{\varphi}\right] \underset{t \rightarrow+\infty}{\longrightarrow} 0
$$

since $\varphi$ has compact support.

In the following, we shall remove the exponent $\varphi$ to simplify the notation. We next set:

$$
\left\{\begin{array}{l}
m_{t}=\varphi\left(g_{0}^{(t)}\right)\left(\frac{\partial}{\partial y} h\left(X_{t}, B_{t}\right) 1_{\left\{X_{t} \geq 0\right\}}-\frac{\partial}{\partial y} h\left(-X_{t},-B_{t}\right) 1_{\left\{X_{t} \leq 0\right\}}\right)+\int_{\mathbb{R}}|z|^{3 / 2} \mathrm{~d} z \int_{0}^{+\infty} \varphi(t+s) \frac{\partial}{\partial y} p_{s}\left(X_{t}, B_{t} ; 0, z\right) \mathrm{d} s \\
n_{t}=\int_{\mathbb{R}}|z|^{3 / 2} \mathrm{~d} z \int_{0}^{+\infty} \varphi(t+s) \frac{\partial}{\partial y} p_{s}\left(X_{t}, B_{t} ; 0, z\right) \mathrm{d} s
\end{array}\right.
$$


Recall from Girsanov's theorem that the process $\left(W_{t}=B_{t}-\int_{0}^{t} \frac{m_{u}}{M_{u}} \mathrm{~d} u, t \geq 0\right)$ is a $\left(\mathbb{Q}_{(x, y)}^{\varphi},\left(\mathcal{F}_{t}\right)\right)$-Brownian motion. Applying Itô's formula, the bracket $\langle Z, W\rangle$ thus equals:

$$
\langle Z, W\rangle_{t}=\left\langle\frac{N}{M}, B\right\rangle_{t}=\left\langle\int_{0}^{\cdot} \frac{n_{u} M_{u}-m_{u} N_{u}}{M_{u}^{2}} d B_{u}, B\right\rangle_{t}=\int_{0}^{t}\left(\frac{n_{u}}{M_{u}}-\frac{m_{u} N_{u}}{M_{u}^{2}}\right) \mathrm{d} u .
$$

Therefore, we may apply Formula (4.6) to obtain the decomposition of $W$ in the filtration $\left(\mathcal{G}_{t}\right)$ :

$$
W_{t}=W_{t}^{\left(g_{0}\right)}+\int_{0}^{t \wedge g_{0}}\left(\frac{n_{u}}{N_{u}}-\frac{m_{u}}{M_{u}}\right) \mathrm{d} u-\int_{t \wedge g_{0}}^{t} \frac{n_{u}-m_{u} \frac{N_{u}}{M_{u}}}{M_{u}-N_{u}} \mathrm{~d} u
$$

where $\left(W_{t}^{\left(g_{0}\right)}, t \geq 0\right)$ is a $\left(\mathbb{Q}_{(x, y)}^{\varphi},\left(\mathcal{G}_{t}\right)\right)$-Brownian motion. Introducing the $\left(\mathbb{P}_{(x, y)},\left(\mathcal{F}_{t}\right)\right)$-Brownian motion $B$, this expression simplifies to:

$$
B_{t}=W_{t}^{\left(g_{0}\right)}+\int_{0}^{t \wedge g_{0}} \frac{n_{u}}{N_{u}} \mathrm{~d} u-\int_{t \wedge g_{0}}^{t} \frac{n_{u}-m_{u}}{M_{u}-N_{u}} \mathrm{~d} u
$$

Therefore, after time $g_{0}$ :

$$
B_{t+g_{0}}=W_{t+g_{0}}^{\left(g_{0}\right)}+\int_{0}^{g_{0}} \frac{n_{u}}{N_{u}} \mathrm{~d} u+\int_{0}^{t} \frac{n_{u+g_{0}}-m_{u+g_{0}}}{N_{u+g_{0}}-M_{u+g_{0}}} \mathrm{~d} u
$$

For $t=0$, we deduce that

$$
B_{g_{0}}=W_{g_{0}}^{\left(g_{0}\right)}+\int_{0}^{g_{0}} \frac{n_{u}}{N_{u}} \mathrm{~d} u
$$

hence,

$$
B_{t+g_{0}}=B_{g_{0}}+\widetilde{W}_{t}^{\left(g_{0}\right)}+\int_{0}^{t} \frac{n_{u+g_{0}}-m_{u+g_{0}}}{N_{u+g_{0}}-M_{u+g_{0}}} \mathrm{~d} u
$$

where $\left(\widetilde{W}_{t}^{\left(g_{0}\right)}=W_{t+g_{0}}^{\left(g_{0}\right)}-W_{g_{0}}^{\left(g_{0}\right)}, t \geq 0\right)$ is a Brownian motion independent from $\mathcal{G}_{g_{0}}$. Point (iv) finally follows from the fact that this (system of) SDEs admits a unique strong solution whose first component never reaches 0.

\section{Penalization With the supremum}

We briefly study in this section the penalization of $X$ by a function of its supremum:

$$
S_{t}=\sup _{u \leq t} X_{u}
$$

Proposition 5.1. Let $\varphi: \mathbb{R} \longmapsto[0,+\infty)$ be a continuous function with compact support. Then the process:

$$
M_{t}^{\varphi}=\varphi\left(S_{t}\right) h\left(S_{t}-X_{t},-B_{t}\right)+\int_{S_{t}}^{+\infty} \varphi(z) \frac{\partial}{\partial z} h\left(z-X_{t},-B_{t}\right) \mathrm{d} z
$$

is a strictly positive and continuous martingale which converges to 0 as $t \longrightarrow+\infty$.

Observe that this martingale is a kind of analogous of Azéma-Yor martingale for the integrated Brownian motion. 
Proof. Assume first that $\varphi$ is differentiable. Since $h$ is harmonic for $\mathcal{G}$, Itô's formula implies that $M$ is a positive and continuous local martingale. Now, let $A$ be a majorant of the support of $\varphi$. If $S_{t} \geq A$, then $M_{t}^{\varphi}=0$. Otherwise, we have, from (2.3):

$$
\begin{aligned}
M_{t}^{\varphi} & \leq\|\varphi\|_{\infty} h\left(S_{t}-X_{t},-B_{t}\right)+\|\varphi\|_{\infty} \int_{S_{t}}^{A} \frac{\partial}{\partial z} h\left(z-X_{t}, B_{t}\right) \mathrm{d} z \\
& \leq\|\varphi\|_{\infty} h\left(A, \sup _{u \leq t}\left(-B_{u}\right)\right) \\
& \leq\|\varphi\|_{\infty}\left(a A^{1 / 6}+b \sqrt{\left|\sup _{u \leq t}\left(-B_{u}\right)\right|}\right)
\end{aligned}
$$

and we deduce that $\left(M_{t}^{\varphi}, t \geq 0\right)$ is a true martingale. The fact that $M^{\varphi}$ converges towards 0 is immediate since $\varphi$ has compact support. We conclude by applying the monotone class theorem to remove the assumption on the differentiability of $\varphi$.

We denote to simplify

$$
\Phi(x, y)=\varphi(x) h(0,-y)+\int_{x}^{+\infty} \varphi(z) \frac{\partial}{\partial z} h(z-x,-y) \mathrm{d} z .
$$

Theorem 5.2. Let $\varphi$ be a continuous function with compact support.

(i) There is the asymptotics:

$$
\mathbb{E}_{(x, y)}\left[\varphi\left(S_{t}\right)\right] \underset{t \rightarrow+\infty}{\sim} \frac{3 \Gamma(1 / 4)}{2^{3 / 4} \pi^{3 / 2} t^{1 / 4}} \Phi(x, y) .
$$

(ii) Let $u \geq 0$ and $(x, y) \in \mathbb{R}^{2}$. For any $\Lambda_{u} \in \mathcal{F}_{u}$, we have:

$$
\lim _{t \rightarrow+\infty} \frac{\mathbb{E}_{(x, y)}\left[1_{\Lambda_{u}} \varphi\left(S_{t}\right)\right]}{\mathbb{E}_{(x, y)}\left[\varphi\left(S_{t}\right)\right]}=\mathbb{E}_{(x, y)}\left[1_{\Lambda_{u}} \frac{M_{u}^{\varphi}}{\Phi(x, y)}\right]
$$

with $\left(M_{u}^{\varphi}, u \geq 0\right)$ the martingale defined in Proposition 5.1.

(iii) There exists a family of probabilities $\left(\mathbb{Q}_{(x, y)}^{\varphi},(x, y) \in \mathbb{R}^{2}\right)$ such that, for any $t \geq 0$ :

$$
\mathbb{Q}_{(x, y) \mid \mathcal{F}_{t}}^{\varphi}=\frac{M_{t}^{\varphi}}{\Phi(x, y)} \cdot \mathbb{P}_{(x, y) \mid \mathcal{F}_{t}} .
$$

(iv) Under $\mathbb{Q}_{(x, y)}^{\varphi}$, the r.v. $S_{\infty}$ is finite and its law is given by:

$$
\mathbb{Q}_{(x, y)}^{\varphi}\left(S_{\infty} \in \mathrm{d} z\right)=\frac{\varphi(x) h(0,-y)}{\Phi(x, y)} \delta_{x}(\mathrm{~d} z)+\varphi(z) \frac{\frac{\partial h}{\partial z}(z-x,-y)}{\Phi(x, y)} 1_{\{z \geq x\}} \mathrm{d} z .
$$

Proof. To prove point (i), we write:

$$
\begin{aligned}
\mathbb{E}_{(x, y)}\left[\varphi\left(S_{t}\right)\right] & =\mathbb{E}_{(x, y)}\left[\varphi\left(S_{t}\right) 1_{\left\{S_{t}>x\right\}}\right]+\mathbb{E}_{(x, y)}\left[\varphi\left(S_{t}\right) 1_{\left\{S_{t}=x\right\}}\right] \\
& =\int_{x}^{+\infty} \varphi(z) \mathbb{P}_{(x, y)}\left(S_{t} \in \mathrm{d} z\right)+\varphi(x) \mathbb{P}_{(x, y)}\left(S_{t}=x\right) \\
& =\int_{x}^{+\infty} \varphi(z) \frac{\partial}{\partial z} \mathbb{P}_{(x, y)}\left(T_{z}>t\right) \mathrm{d} z+\varphi(x) \mathbb{P}_{(0,-y)}\left(T_{0}>t\right) 1_{\{y<0\}} \\
& =\int_{x}^{+\infty} \varphi(z) \frac{\partial}{\partial z} \mathbb{P}_{(z-x,-y)}\left(T_{0}>t\right) \mathrm{d} z+\varphi(x) \mathbb{P}_{(0,-y)}\left(T_{0}>t\right) 1_{\{y<0\}} \\
& \underset{t \rightarrow+\infty}{\sim} \frac{3 \Gamma(1 / 4)}{2^{3 / 4} \pi^{3 / 2} t^{1 / 4}}\left(\int_{x}^{+\infty} \varphi(z) \frac{\partial}{\partial z} h(z-x,-y) \mathrm{d} z+\varphi(x) h(0,-y)\right) .
\end{aligned}
$$


Then, for $t>u$, from the Markov property and since $S_{u} \geq X_{u}$ :

$$
\begin{aligned}
\mathbb{E}_{(x, y)}\left[\varphi\left(S_{t}\right) \mid \mathcal{F}_{u}\right] & =\mathbb{E}_{(x, y)}\left[\varphi\left(S_{u} \vee \sup _{u \leq s \leq t} X_{s}\right) \mid \mathcal{F}_{u}\right] \\
& =\widehat{\mathbb{E}}_{\left(X_{u}, B_{u}\right)}\left[\varphi\left(S_{u} \vee \widehat{S}_{t-u}\right)\right]
\end{aligned}
$$

(where $\widehat{\mathbb{E}}$ and $\widehat{S}$ are associated with a copy of $X$ which is independent from $\mathcal{F}_{u}$ )

$$
\begin{aligned}
& \underset{t \rightarrow+\infty}{\sim} \frac{3 \Gamma(1 / 4)}{2^{3 / 4} \pi^{3 / 2} t^{1 / 4}}\left(\int_{X_{u}}^{+\infty} \varphi\left(S_{u} \vee z\right) \frac{\partial}{\partial z} h\left(z-X_{u},-B_{u}\right) \mathrm{d} z+\varphi\left(S_{u} \vee X_{u}\right) h\left(0,-B_{u}\right)\right) \\
& \underset{t \rightarrow+\infty}{\sim} \frac{3 \Gamma(1 / 4)}{2^{3 / 4} \pi^{3 / 2} t^{1 / 4}}\left(\varphi\left(S_{u}\right) h\left(S_{u}-X_{u},-B_{u}\right)+\int_{S_{u}}^{+\infty} \varphi(z) \frac{\partial}{\partial z} h\left(z-X_{u},-B_{u}\right) \mathrm{d} z\right) .
\end{aligned}
$$

Therefore, points (ii) and (iii) follow from Theorem 2.3.

To compute the law of $S_{\infty}$ under $\mathbb{Q}_{(x, y)}^{\varphi}$, observe that, for $c>x$ :

$$
\begin{aligned}
\mathbb{Q}_{(x, y)}^{\varphi}\left(S_{t}>c\right)=\mathbb{Q}_{(x, y)}^{\varphi}\left(T_{c}<t\right)= & \mathbb{E}_{(x, y)}\left[\int_{c}^{+\infty} \varphi(z) \frac{\partial}{\partial z} h\left(z-c,-B_{T_{c}}\right) \mathrm{d} z 1_{\left\{T_{c}<t\right\}}\right] / \Phi(x, y) \\
& \underset{t \rightarrow+\infty}{\longrightarrow} \mathbb{E}_{(x, y)}\left[\int_{c}^{+\infty} \varphi(z) \frac{\partial}{\partial z} h\left(z-c,-B_{T_{c}}\right) \mathrm{d} z\right] / \Phi(x, y)
\end{aligned}
$$

from the monotone convergence theorem. Then, applying Fubini and exchanging the derivative and the expectation:

$$
\mathbb{Q}_{(x, y)}^{\varphi}\left(S_{\infty}>c\right)=\frac{1}{\Phi(x, y)} \int_{c}^{+\infty} \varphi(z) \frac{\partial}{\partial z} \mathbb{E}_{(x, y)}\left[h\left(z-c,-B_{T_{c}}\right)\right] \mathrm{d} z
$$

Observe now that, by symmetry and translation:

$$
\mathbb{E}_{(x, y)}\left[h\left(z-c,-B_{T_{c}}\right)\right]=\mathbb{E}_{(z-x,-y)}\left[h\left(z-c, B_{T_{z-c}}\right)\right]
$$

hence, from Lemma 2.9,

$$
\mathbb{E}_{(x, y)}\left[h\left(z-c,-B_{T_{c}}\right)\right]=h(z-x,-y)-h(c-x,-y)
$$

which proves point (iv).

\section{Appendix: Proof of Proposition 3.1}

We prove in this section the following asymptotics formula (Prop. 3.1) for the survival function of the $n$th passage time at level 0:

$$
\mathbb{P}_{(0, b)}\left(T_{0}^{(n)}>t\right) \underset{t \rightarrow+\infty}{\sim} \frac{2^{1 / 4} \Gamma(1 / 4) \sqrt{|b|}}{\sqrt{\pi}(n-1) !}\left(\frac{9}{4 \pi^{2}}\right)^{n / 2} \frac{(\ln (t))^{n-1}}{t^{1 / 4}}
$$

where $n \geq 1$ and $b \neq 0$.

Proof. Observe first that for $n=1$, Proposition 3.1 agrees with Theorem 2.1, so we now assume that $n \geq 2$. Suppose that $b>0$ for simplicity. From Lachal ([19], Thm. 1), we have:

$$
\mathbb{P}_{(0, b)}\left(T_{0}^{(n)} \in \mathrm{d} t, \frac{\left|B_{T_{0}^{(n)}}\right|}{\sqrt{t}} \in \mathrm{d} z\right) /(\mathrm{d} t \mathrm{~d} z)=\frac{1}{\pi^{2} b \sqrt{t}} \mathrm{e}^{-\frac{2}{t} b^{2}-2 z^{2}} \int_{0}^{+\infty} K_{i \gamma}\left(\frac{4 b z}{\sqrt{t}}\right) \frac{\gamma \sinh (\pi \gamma)}{\left(2 \cosh \left(\frac{\pi \gamma}{3}\right)\right)^{n}} \mathrm{~d} \gamma
$$


where $K_{\nu}$ denotes MacDonald function with index $\nu$ (see [2], p. 374). Now, the integral may be decomposed in:

$$
\begin{aligned}
& \int_{0}^{+\infty} K_{i \gamma}\left(\frac{4 b z}{\sqrt{t}}\right) \frac{\gamma \sinh (\pi \gamma)}{\left(2 \cosh \left(\frac{\pi \gamma}{3}\right)\right)^{n}} \mathrm{~d} \gamma \\
& =\frac{1}{2^{n}} \int_{0}^{+\infty} \gamma K_{i \gamma}\left(\frac{4 b z}{\sqrt{t}}\right) \frac{4 \cosh ^{2}\left(\frac{\pi \gamma}{3}\right) \sinh \left(\frac{\pi \gamma}{3}\right)-\sinh \left(\frac{\pi \gamma}{3}\right)}{\left(\cosh \left(\frac{\pi \gamma}{3}\right)\right)^{n}} \mathrm{~d} \gamma \\
& =\frac{1}{2^{n-2}} \int_{0}^{+\infty} \gamma K_{i \gamma}\left(\frac{4 b z}{\sqrt{t}}\right) \frac{\sinh \left(\frac{\pi \gamma}{3}\right)}{\left(\cosh \left(\frac{\pi \gamma}{3}\right)\right)^{n-2}} \mathrm{~d} \gamma-\frac{1}{2^{n}} \int_{0}^{+\infty} \gamma K_{i \gamma}\left(\frac{4 b z}{\sqrt{t}}\right) \frac{\sinh \left(\frac{\pi \gamma}{3}\right)}{\left(\cosh \left(\frac{\pi \gamma}{3}\right)\right)^{n}} \mathrm{~d} \gamma
\end{aligned}
$$

so we need to estimate:

$$
\int_{0}^{+\infty} \gamma K_{i \gamma}\left(\frac{4 b z}{\sqrt{t}}\right) \frac{\sinh \left(\frac{\pi \gamma}{3}\right)}{\left(\cosh \left(\frac{\pi \gamma}{3}\right)\right)^{k}} \mathrm{~d} \gamma \quad(k \in \mathbb{N})
$$

Assume, for the moment, that the following asymptotics holds:

Lemma A.1. For $k \geq 0$ :

$$
\int_{0}^{+\infty} \gamma K_{i \gamma}\left(\frac{4 b z}{\sqrt{t}}\right) \frac{\sinh \left(\frac{\pi \gamma}{3}\right)}{\left(\cosh \left(\frac{\pi \gamma}{3}\right)\right)^{k}} \mathrm{~d} \gamma \underset{t \rightarrow+\infty}{=} \frac{\alpha_{k}}{\sqrt{t}}-\beta_{k} \frac{(\ln (t))^{k-1}}{t^{3 / 4}}+\mathrm{o}\left(\frac{(\ln (t))^{k-1}}{t^{3 / 4}}\right)
$$

with

$$
\beta_{0}=0 \quad \text { and, for } k \geq 1, \quad \beta_{k}=\frac{2^{k} 9 \sqrt{2}}{\sqrt{\pi}(k-1) !}\left(\frac{9}{4 \pi^{2}}\right)^{k / 2-1} z^{3 / 2} b \sqrt{b} .
$$

Going back to (A.1), we obtain:

$$
\begin{array}{r}
\mathbb{P}_{(0, b)}\left(T_{0}^{(n)} \in \mathrm{d} t, \frac{\left|B_{T_{0}^{(n)}}\right|}{\sqrt{t}} \in \mathrm{d} z\right) /(\mathrm{d} z \mathrm{~d} t) \\
\underset{\substack{t \rightarrow+\infty \\
=}}{=} \frac{\mathrm{e}^{-2 z^{2}}}{\pi^{2} b \sqrt{t}}\left(\frac{1}{\sqrt{t}}\left(\frac{\alpha_{n-2}}{2^{n-2}}-\frac{\alpha_{n}}{2^{n}}\right)+\frac{\beta_{n}}{2^{n}} \frac{(\ln (t))^{n-1}}{t^{3 / 4}}+\mathrm{o}\left(\frac{(\ln (t))^{n-1}}{t^{3 / 4}}\right)\right) .
\end{array}
$$

But, since:

$$
\int_{0}^{+\infty} \int_{0}^{+\infty} \mathbb{P}_{(0, b)}\left(T_{0}^{(n)} \in \mathrm{d} t, \frac{\left|B_{T_{0}^{(n)}}\right|}{\sqrt{t}} \in \mathrm{d} z\right)=1
$$

we must have $\frac{\alpha_{n-2}}{2^{n-2}}-\frac{\alpha_{n}}{2^{n}}=0$ for this function to be integrable with respect to $t$, so it remains

$$
\mathbb{P}_{(0, b)}\left(T_{0}^{(n)} \in \mathrm{d} t, \frac{\left|B_{T_{0}^{(n)}}\right|}{\sqrt{t}} \in \mathrm{d} z\right) /(\mathrm{d} t \mathrm{~d} z) \underset{t \rightarrow+\infty}{\sim} \frac{4 \sqrt{2 b}}{\sqrt{\pi}(n-1) !}\left(\frac{9}{4 \pi^{2}}\right)^{n / 2} \frac{(\ln (t))^{n-1}}{t^{5 / 4}} \mathrm{e}^{-2 z^{2}} z^{3 / 2}
$$

and, finally, integrating with respect to $z$ :

$$
\begin{aligned}
\mathbb{P}_{(0, b)}\left(T_{0}^{(n)} \in \mathrm{d} t\right) / \mathrm{d} t \underset{t \rightarrow+\infty}{\sim} \frac{4 \sqrt{2 b}}{\sqrt{\pi}(n-1) !}\left(\frac{9}{4 \pi^{2}}\right)^{n / 2} \frac{(\ln (t))^{n-1}}{t^{5 / 4}} \int_{0}^{+\infty} \mathrm{e}^{-2 z^{2}} z^{3 / 2} \mathrm{~d} z, \\
\underset{t \rightarrow+\infty}{\sim} \frac{4 \sqrt{2 b}}{\sqrt{\pi}(n-1) !}\left(\frac{9}{4 \pi^{2}}\right)^{n / 2} \frac{(\ln (t))^{n-1}}{t^{5 / 4}} \frac{\Gamma(1 / 4)}{2^{4+1 / 4}}
\end{aligned}
$$

and the result follows by integration with respect to $t$. 
Therefore, it only remains to prove Lemma A.1. We shall distinguish between three cases. In the following, to simplify the notation, we set:

$$
a=\frac{4 b z}{\sqrt{t}}
$$

- First, assume that $k \geq 2$, so that all the integrals we are going to write are absolutely convergent. From the integral expression

$$
K_{i \gamma}(a)=\int_{0}^{+\infty} \mathrm{e}^{-a \cosh (t)} \cos (\gamma t) \mathrm{d} t=a \int_{0}^{+\infty} \sinh (u) \mathrm{e}^{-a \cosh (u)} \frac{\sin (\gamma u)}{\gamma} \mathrm{d} u
$$

we obtain, applying Fubini and integrating by parts with respect to $\gamma$ :

$$
\begin{aligned}
\int_{0}^{+\infty} \gamma K_{i \gamma}(a) \frac{\sinh \left(\frac{\pi \gamma}{3}\right)}{\left(\cosh \left(\frac{\pi \gamma}{3}\right)\right)^{k}} \mathrm{~d} \gamma & =\int_{0}^{+\infty} a \sinh (u) \mathrm{e}^{-a \cosh (u)} \mathrm{d} u \int_{0}^{+\infty} \sin (\gamma u) \frac{\sinh \left(\frac{\pi \gamma}{3}\right)}{\left(\cosh \left(\frac{\pi \gamma}{3}\right)\right)^{k}} \mathrm{~d} \gamma \\
& =\frac{3 a}{\pi(k-1)} \int_{0}^{+\infty} u \sinh (u) \mathrm{e}^{-a \cosh (u)} \mathrm{d} u \int_{0}^{+\infty} \frac{\cos (\gamma u)}{\left(\cosh \left(\frac{\pi \gamma}{3}\right)\right)^{k-1}} \mathrm{~d} \gamma
\end{aligned}
$$

and this last expression is a cosinus transform which may be found, for instance, in Erdelyi [6], page $30^{2}$.

1) if $k=2 p$ with $p \geq 1$ :

$$
\int_{0}^{+\infty} \frac{\cos (\gamma u)}{\left(\cosh \left(\frac{\pi \gamma}{3}\right)\right)^{2 p-1}} \mathrm{~d} \gamma=\frac{2^{2 p-3}}{(2(p-1)) !} \frac{3}{\cosh \left(\frac{3}{2} u\right)} \prod_{r=1}^{p-1}\left(\frac{9 u^{2}}{4 \pi^{2}}+\left(r-\frac{1}{2}\right)^{2}\right)
$$

2) if $k=2 p+1$ with $p \geq 1$ :

$$
\int_{0}^{+\infty} \frac{\cos (\gamma u)}{\left(\cosh \left(\frac{\pi \gamma}{3}\right)\right)^{2 p}} \mathrm{~d} \gamma=\frac{4^{p-1}}{2 \pi(2 p-1) !} \frac{9 u}{\sinh \left(\frac{3}{2} u\right)} \prod_{r=1}^{p-1}\left(\frac{9 u^{2}}{4 \pi^{2}}+r^{2}\right) .
$$

To continue the computation, we set:

$$
\Gamma_{k-1}(u)=\frac{3 u}{\pi(k-1)} \int_{0}^{+\infty} \frac{\cos (\gamma u)}{\left(\cosh \left(\frac{\pi \gamma}{3}\right)\right)^{k-1}} \mathrm{~d} \gamma
$$

Then, the change of variable $v=\cosh (u)-1$ leads to:

$$
\int_{0}^{+\infty} \gamma K_{i \gamma}(a) \frac{\sinh \left(\frac{\pi \gamma}{3}\right)}{\left(\cosh \left(\frac{\pi \gamma}{3}\right)\right)^{k}} \mathrm{~d} \gamma=a \mathrm{e}^{-a} \int_{0}^{+\infty} \mathrm{e}^{-a v} \Gamma_{k-1}(\operatorname{Argcosh}(1+v)) \mathrm{d} v
$$

and another integration by parts yields:

$$
\int_{0}^{+\infty} \gamma K_{i \gamma}(a) \frac{\sinh \left(\frac{\pi \gamma}{3}\right)}{\left(\cosh \left(\frac{\pi \gamma}{3}\right)\right)^{k}} \mathrm{~d} \gamma=a \mathrm{e}^{-a} C_{k-1}-a^{2} \mathrm{e}^{-a} \int_{0}^{+\infty} \mathrm{e}^{-a v} \mathrm{~d} v \int_{v}^{+\infty} \Gamma_{k-1}(\operatorname{Arg} \cosh (1+s)) \mathrm{d} s
$$

where

$$
C_{k-1}=\int_{0}^{+\infty} \Gamma_{k-1}(\operatorname{Argcosh}(1+s)) \mathrm{d} s
$$

\footnotetext{
${ }^{2}$ By convention, we set $\prod_{r=1}^{0}=1$.
} 
To obtain the asymptotics of the last integral, we shall apply the Tauberian's Theorem ([7], Chap. XIII-5, p. 446). Letting $v$ tend toward $+\infty$, we obtain, thanks to (A.2) and (A.3), the following asymptotics:

1) if $k=2 p$ with $p \geq 1$ :

$$
\Gamma_{2 p-1}(\operatorname{Argcosh}(1+v)) \underset{v \rightarrow+\infty}{\sim} \frac{3}{\pi(2 p-1)} \frac{2^{2 p-2}}{(2(p-1)) !} \frac{3}{(2 v)^{3 / 2}}\left(\frac{9}{4 \pi^{2}}\right)^{p-1}(\ln (v))^{2 p-1}
$$

2) if $k=2 p+1$ with $p \geq 1$ :

$$
\Gamma_{2 p}(\operatorname{Argcosh}(1+v)) \underset{v \rightarrow+\infty}{\sim} \frac{3}{\pi(2 p)} \frac{4^{p-1}}{\pi(2 p-1) !} \frac{9}{(2 v)^{3 / 2}}\left(\frac{9}{4 \pi^{2}}\right)^{p-1}(\ln (v))^{2 p}
$$

and these two formulae reduce to:

$$
\Gamma_{k-1}(\operatorname{Argcosh}(1+v)) \underset{v \rightarrow+\infty}{\sim} \frac{9}{\pi(k-1) !} \frac{2^{k-2}}{(2 v)^{3 / 2}}\left(\frac{9}{4 \pi^{2}}\right)^{k / 2-1}(\ln (v))^{k-1},
$$

hence, integrating

$$
\int_{v}^{+\infty} \Gamma_{k-1}(\operatorname{Argcosh}(1+s)) \mathrm{d} s \underset{v \rightarrow+\infty}{\sim} \frac{9}{\pi(k-1) !} \frac{2^{k-2}}{\sqrt{2 v}}\left(\frac{9}{4 \pi^{2}}\right)^{k / 2-1}(\ln (v))^{k-1},
$$

and the Tauberian's theorem gives:

$$
\int_{0}^{+\infty} \mathrm{e}^{-a v} \int_{v}^{+\infty} \Gamma_{k-1}(\operatorname{Arg} \cosh (1+s)) \mathrm{d} s \underset{a \rightarrow 0}{\sim} \Gamma(1 / 2) \frac{9}{\pi(k-1) !} \frac{2^{k-2}}{\sqrt{2 a}}\left(\frac{9}{4 \pi^{2}}\right)^{k / 2-1}(-\ln (a))^{k-1} .
$$

Therefore, we deduce that:

$$
\begin{aligned}
& \int_{0}^{+\infty} \gamma K_{i \gamma}(a) \frac{\sinh \left(\frac{\pi \gamma}{3}\right)}{\left(\cosh \left(\frac{\pi \gamma}{3}\right)\right)^{k}} \mathrm{~d} \gamma \\
& \qquad \underset{a \rightarrow 0}{=} a C_{k-1}-\frac{9 a^{2}}{\sqrt{\pi}(k-1) !} \frac{2^{k-2}}{\sqrt{2 a}}\left(\frac{9}{4 \pi^{2}}\right)^{k / 2-1}(-\ln (a))^{k-1}+\mathrm{o}\left(a^{3 / 2}(-\ln (a))^{k-1}\right)
\end{aligned}
$$

which is the expression given in Lemma A.1 after replacing $a$ by $\frac{4 b z}{\sqrt{t}}$.

- When $k=1$, we rather write, integrating by parts:

$$
\begin{aligned}
\int_{0}^{+\infty} \gamma K_{i \gamma}(a) \frac{\sinh \left(\frac{\pi \gamma}{3}\right)}{\cosh \left(\frac{\pi \gamma}{3}\right)} \mathrm{d} \gamma & =\int_{0}^{+\infty}\left(a \int_{0}^{+\infty} \sinh (u) \mathrm{e}^{-a \cosh (u)} \sin (\gamma u) \mathrm{d} u\right) \tanh \left(\frac{\pi \gamma}{3}\right) \mathrm{d} \gamma \\
& =\frac{\pi a}{3} \int_{0}^{+\infty} \frac{\sinh (u)}{u} \mathrm{e}^{-a \cosh (u)} \mathrm{d} u \int_{0}^{+\infty} \frac{\cos (\gamma u)}{\left(\cosh \left(\frac{\pi \gamma}{3}\right)\right)^{2}} \mathrm{~d} \gamma \\
& =\frac{\pi a}{3} \int_{0}^{+\infty} \frac{\sinh (u)}{u} \mathrm{e}^{-a \cosh (u)} \frac{9}{2 \pi} \frac{u}{\sinh \left(\frac{3}{2} u\right)} \mathrm{d} u \quad \text { (thanks to (A.3)) } \\
& =\frac{3 a}{2} \mathrm{e}^{-a} \int_{0}^{+\infty} \mathrm{e}^{-a v} \frac{1}{\sinh \left(\frac{3}{2} \operatorname{Arg} \cosh (1+v)\right)} \mathrm{d} v \\
& =\frac{3 a}{2} \mathrm{e}^{-a} C_{0}-\frac{3 a^{2}}{2} \mathrm{e}^{-a} \int_{0}^{+\infty} \mathrm{e}^{-a v}\left(\int_{v}^{+\infty} \frac{1}{\sinh \left(\frac{3}{2} \operatorname{Arg} \cosh (1+s)\right)} \mathrm{d} s\right) \mathrm{d} v
\end{aligned}
$$


where $C_{0}=\int_{0}^{+\infty} \frac{1}{\sinh \left(\frac{3}{2} \operatorname{Arg} \cosh (1+v)\right)} \mathrm{d} v$ and, as before, we obtain the asymptotics:

$$
\int_{0}^{+\infty} \gamma K_{i \gamma}(a) \frac{\sinh \left(\frac{\pi \gamma}{3}\right)}{\cosh \left(\frac{\pi \gamma}{3}\right)} \mathrm{d} \gamma \underset{a \rightarrow 0}{=} \frac{3 a}{2} C_{0}-\frac{3 \sqrt{\pi}}{\sqrt{2}} a^{3 / 2}+o\left(a^{3 / 2}\right) .
$$

- When $k=0$, we shall compute explicitly the expression:

$$
f(a):=\int_{0}^{+\infty} \gamma K_{i \gamma}(a) \sinh \left(\frac{\pi \gamma}{3}\right) \mathrm{d} \gamma=\int_{0}^{+\infty} \frac{\pi^{2} \sinh \left(\frac{\pi \gamma}{3}\right)}{2 \sinh (\pi \gamma)} K_{i \gamma}(a) \frac{2}{\pi^{2}} \gamma \sinh (\pi \gamma) \mathrm{d} \gamma .
$$

Indeed, applying the Lebedev transform pair:

$$
\left\{\begin{array}{l}
\widehat{f}(\gamma)=\int_{0}^{+\infty} f(a) K_{i \gamma}(a) \frac{\mathrm{d} a}{a} \\
f(a)=\int_{0}^{+\infty} \widehat{f}(\gamma) K_{i \gamma}(a) \frac{2}{\pi^{2}} \gamma \sinh (\pi \gamma) \mathrm{d} \gamma
\end{array}\right.
$$

we deduce that:

$$
\begin{aligned}
\frac{\pi^{2}}{2} \frac{\sinh \left(\frac{\pi \gamma}{3}\right)}{\sinh (\pi \gamma)} & =\int_{0}^{+\infty} f(a) K_{i \gamma}(a) \frac{\mathrm{d} a}{a} \\
& =\int_{0}^{+\infty} \cos (\gamma u) \mathrm{d} u \int_{0}^{+\infty} \mathrm{e}^{-a \cosh (u)} f(a) \frac{\mathrm{d} a}{a}
\end{aligned}
$$

hence, from the injectiveness of the cosine transform, see Erdelyi [6], p. 30 Formula (6):

$$
\frac{\pi}{2 \sin (\pi / 3)} \frac{1}{\cosh (u)+\cos (\pi / 3)}=\int_{0}^{+\infty} \mathrm{e}^{-a \cosh (u)} f(a) \frac{\mathrm{d} a}{a}
$$

and the injectiveness of the Laplace transform finally gives:

$$
f(a)=\frac{\pi a}{\sqrt{3}} \mathrm{e}^{-\frac{a}{2}}
$$

Acknowledgements. We wish to thank the two anonymous referees whose very careful reading and detailed reviews greatly improved the final version of the paper.

\section{REFERENCES}

[1] M. Abramowitz and I.A. Stegun, Handbook of Mathematical Functions with Formulas, Graphs, and Mathematical Tables. Reprint of the 1972 edition. Dover Publications Inc., New York (1992).

[2] F. Aurzada and T. Simon, Persistence probabilities and exponents. Preprint arXiv:1203.6554 (2012).

[3] J. Bertoin, Reflecting a Langevin process at an absorbing boundary. Ann. Probab. 35 (2007) 2021-2037.

[4] M. Bossy and J.-F. Jabir, On confined McKean Langevin processes satisfying the mean no-permeability boundary condition. Stoch. Process. Appl. 121 (2011) 2751-2775.

[5] P. Debs, Penalisation of the standard random walk by a function of the one-sided maximum, of the local time, or of the duration of the excursions, in Séminaire de probabilités XLII. Vol. 1979, Lect. Notes Math. Springer, Berlin (2009) 331-363.

[6] A. Erdélyi, W. Magnus, F. Oberhettinger and F.G. Tricomi, Tables of integral transforms. McGraw-Hill Book Company, Inc., New York-Toronto-London (1954), Vol. I. Based, in part, on notes left by Harry Bateman.

[7] W. Feller, An introduction to probability theory and its applications. Second edition. John Wiley \& Sons Inc., New York (1971), Vol. II.

[8] P. Groeneboom, G. Jongbloed and J.A. Wellner, Integrated Brownian motion, conditioned to be positive. Ann. Probab. 27 (1999) 1283-1303.

[9] M. Goldman, On the first passage of the integrated Wiener process. Ann. Mat. Statist. 42 (1971) 2150-2155. 
[10] E. Jacob, Processus de Langevin réfléchis au second ordre. Ph.D. thesis, Université Pierre et Marie Curie, Paris VI (2010).

[11] Y. Isozaki and S. Kotani, Asymptotic estimates for the first hitting time of fluctuating additive functionals of Brownian motion. In Séminaire de Probabilités, XXXIV. Vol. 1729 of Lect. Notes Math. Springer, Berlin (2000) 374-387.

[12] I. Karatzas and S.E. Shreve, Brownian motion and stochastic calculus, 2nd edition. Vol. 113 of Grad. Texts Math. SpringerVerlag, New York (1991).

[13] D. Khoshnevisan and Z. Shi, Chung's law for integrated Brownian motion. Trans. Amer. Math. Soc. 350 (1998) $4253-4264$.

[14] A. Lachal, Sur l'intégrale du mouvement brownien. C. R. Acad. Sci. Paris Sér. I Math. 311 (1990) $461-464$.

[15] A. Lachal, Sur le premier instant de passage de l'intégrale du mouvement brownien. Ann. Inst. Henri Poincaré Probab. Statist. 27 (1991) 385-405.

[16] A. Lachal, Sur les excursions de l'intégrale du mouvement brownien. C. R. Acad. Sci. Paris Sér. I Math. 314 (1992) $1053-1056$.

[17] A. Lachal, Dernier instant de passage pour l'intégrale du mouvement brownien. Stochastic Process. Appl. 49 (1994) 57-64.

[18] A. Lachal, Sur les temps de passages successifs de l'intégrale du mouvement brownien. C. R. Acad. Sci. Paris Sér. I Math. 321 (1995) 903-908.

[19] A. Lachal, Les temps de passage successifs de l'intégrale du mouvement brownien. Ann. Inst. Henri Poincaré Probab. Statist. 33 (1997) 1-36.

[20] A. Lachal, Application de la théorie des excursions à l'intégrale du mouvement brownien. In Séminaire de Probabilités XXXVII. Vol. 1832 of Lect. Notes Math. Springer, Berlin (2003) 109-195.

[21] H.P. McKean, Jr. A winding problem for a resonator driven by a white noise. J. Math. Kyoto Univ. 2 (1963) $227-235$.

[22] R. Mansuy and M. Yor, Random times and enlargements of filtrations in a Brownian setting. Vol. 1873 of Lect. Notes Math. Springer-Verlag, Berlin (2006).

[23] J. Najnudel, B. Roynette and M. Yor, A global view of Brownian penalisations. Vol. 19 of MSJ Memoirs. Mathematical Society of Japan, Tokyo (2009).

[24] C. Profeta, Penalizing null recurrent diffusions. Electron. J. Probab. 17 (2012) 23.

[25] B. Roynette, P. Vallois and M. Yor, Limiting laws associated with Brownian motion perturbed by its maximum, minimum and local time II. Studia Sci. Math. Hungar. 43 (2006) 295-360.

[26] B. Roynette, P. Vallois and M. Yor, Some penalisations of the Wiener measure. Jpn. J. Math. 1 (2006) $263-290$.

[27] B. Roynette and M. Yor, Penalising Brownian paths. Vol. 1969 of Lect. Notes Math. Springer-Verlag, Berlin (2009).

[28] P. Salminen and P. Vallois, On subexponentiality of the Lévy measure of the diffusion inverse local time; with applications to penalizations. Electron. J. Probab. 14 (2009) 1963-1991.

[29] J. Touboul and O. Faugeras, A characterization of the first hitting time of double integral processes to curved boundaries. Adv. Appl. Probab. 40 (2008) 501-528.

[30] K. Yano, Y. Yano and M. Yor, Penalisation of a stable Lévy process involving its one-sided supremum. Ann. Inst. Henri Poincaré Probab. Stat. 46 (2010) 1042-1054. 